Article

\title{
Gradient Heatmetry Advances
}

\author{
Sergey Z. Sapozhnikov ${ }^{1}$, Vladimir Y. Mityakov ${ }^{1}$, Andrey V. Mityakov ${ }^{1, *}$, Andrey A. Gusakov ${ }^{1}$, \\ Elza R. Zainullina ${ }^{1}$, Mikhail A. Grekov ${ }^{1}$, Vladimir V. Seroshtanov ${ }^{1, *}$, Alexander Bashkatov ${ }^{2} \mathbb{D}$, \\ Alexander Y. Babich ${ }^{1}$ and Andrey V. Pavlov ${ }^{1}$ \\ 1 Science Educational Centre "Energy Thermophysics", Peter the Great St.Petersburg Polytechnic University \\ (SPbPU), St.Petersburg 195251, Russia; serg.sapozhnikov@mail.ru (S.Z.S.); mitvlad@mail.ru (V.Y.M.); \\ gusakov@jsti.spbstu.ru (A.A.G.); zajnullina_er@spbstu.ru (E.R.Z.); grekov_ma@spbstu.ru (M.A.G.); \\ babich_ayu@spbstu.ru (A.Y.B.); pavlov.av@spbstu.ru (A.V.P.) \\ 2 Institute of Fluid Dynamics, Helmholtz-Zentrum Dresden-Rossendorf, 01328 Dresden, Germany; \\ a.bashkatov@hzdr.de \\ * Correspondence: mitiakov@spbstu.ru (A.V.M.); serosht_vv@spbstu.ru (V.V.S.); \\ Tel.: +7-921-590-7421 (A.V.M.); +7-812-552-7773 (V.V.S.)
}

Received: 22 September 2020; Accepted: 19 November 2020; Published: 25 November 2020

check for updates

\begin{abstract}
The paper describes a unique method of heat flux measurement, i.e., gradient heatmetry. Gradient heatmetry is performed using gradient heat flux sensors (GHFS) developed on the anisotropic thermoelements basis. The principle of GHFS' operation leads to the fact that their response time is about $10 \mathrm{~ns}$, and the volt-watt sensitivity does not depend on the thickness. GHFS are compared with other types heat flux sensors, with the GHFS features depending on the materials being described. The theory and examples of gradient heatmetry applications in thermophysical experiment are provided.
\end{abstract}

Keywords: gradient heat flux sensor; heat flux; heat transfer; calibration; convection; condensation; boiling

\section{Introduction}

Direct heat flux measurement is one of the most important tasks in thermal physics, power engineering, metalworking, power electronics, etc. This kind of measurement is important for industry development. Heat flux recording devices have become widely used in laboratory experiments (study of convective heat transfer [1], and heat transfer during phase transition [2,3]), technology (boiler plants, nuclear power plants, electric machines [4]) and everyday living (climate control, energy efficiency of buildings and structures [5]). Monitoring and control systems based on heat flux measurement [6] are of particular interest.

Despite the growing demand of heat flux measurement (heatmetry), there are few types of heat flux sensors (HFS) in the world. This paper describes surface-mounted heat flux sensors, whose the sensitive element is a plate with the smallest face placed on an isothermal surface [7,8]. A HFS generates electrical signals with magnitude and direction associated with the heat flux per unit area vector passing through the sensor.

The vast majority of HFS have an upper temperature limit of 300...500 K [9] due to the sensors' materials. Such sensors can not be used for high-temperature measurements in the power industry, metallurgy, etc. The majority of surface-mounted HFS consist of plates with differential thermocouple junctions on both surfaces. The thermocouple is usually made as a battery of hundreds of junctions connected in series to amplify the signal (multijunction thermocouple). Thermocouple-type HFS' vectors of temperature gradient and thermal EMF $\mathrm{E}_{\|}(\mathrm{V} / \mathrm{m})$ are collinear. 
As a result of research [10-12] at the Peter the Great Saint Petersburg Polytechnic University since 1996, fundamentally different HFS have been developed and created. They are based on the anisotropic thermoelements (ATE), which have anisotropy of the following properties: thermal conductivity, electrical conductivity and thermal EMF. The temperature gradient of such an HFS has two components: along the applied heat flux per unit area vector $q_{0}$ and normal to it [13]. This phenomenon is called the transverse Seebeck effect. The electric field intensity vector $E_{\perp}$ is proportional to the transverse temperature gradient and normal to the vector $q_{0}$. Since this HFS type generates an output signal proportional to the transverse temperature gradient, they have been called gradient heat flux sensors (GHFS) [14]. A GHFS generates an electrical signal in a very thin surface layer of the sensor, resulting in a response time of $10^{-8}-10^{-9}$ seconds. A fast response time expands the possibilities of the thermophysical experiment, making measurements almost inertia-free.

The number of materials with natural anisotropy that can be used as ATE is limited. Such materials include antimony cadmium, single-crystalline bismuth, single-crystalline higher manganese silicide and others [14]. The melting point of most anisotropic metals is about 500 . 6 600; therefore, GHFS have a narrow operating temperature range. Said shortcomings notwithstanding, there are examples of successful applications of GHFS based on single-crystalline bismuth in the study of convective heat transfer.

Thermal and electrical anisotropy can also be obtained synthetically [14]. A synthetic GHFS is a system of alternating layers of two materials (copper-constantan, chromel-copel, etc.) with different thermal and electrophysical properties [14]. Since such sensors consist of separate macrostructures, we call them heterogeneous GHFS (HGHFS). Otherwise, the principle of operation of GHFS and HGHFS is identical, but the operating temperature range of a HGHFS is much wider: working resistance of HGHFS can reach $1300 \mathrm{~K}$ and higher.

\section{GHFS Principle of Operation}

The GHFS' operation is based on the generation of transverse thermal EMF in the anisotropic medium. Figure 1 shows the ATE as a parallelepiped, where $l>b>>a$. Note that all thermal and electrophysical properties are tensor values due to anisotropy [10]. If crystallographic axes coincide with an arbitrarily given (laboratory) coordinate system, heat-conduction and thermal EMF tensors are diagonal. We have chosen a coordinate system in where the $y$-axis and the $C_{2}$ crystallographic axis coincide.

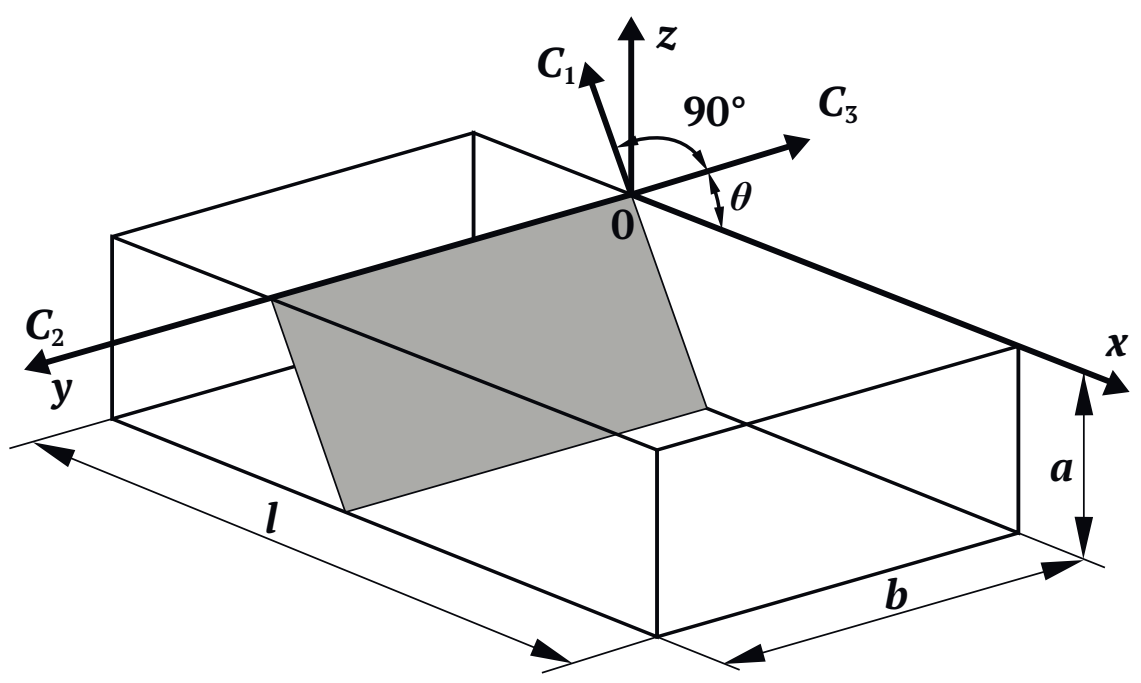

Figure 1. Anisotropic thermoelements: $l, b$ and $a$ are length, width and height, respectively; $C_{1}, C_{2}$ and $C_{3}$ are crystallographic anisotropic thermoelement (ATE) axes; $x, y$ and $z$ are a Cartesian coordinate system. 
The target function is the volt-watt sensitivity $S_{0}$ of the ATE under consideration. The ATE sensitivity must be linked to the generated signal, the external heat flux per unit area and the ATE plan-area:

$$
S_{0}=\frac{E}{q_{0} A}
$$

where $E(\mathrm{~V})$ is the thermal EMF generated by ATE; $q_{0}\left(\mathrm{~W} / \mathrm{m}^{2}\right)$ is external heat flux per unit area; $A=l \times b\left(\mathrm{~m}^{2}\right)$ is ATE plan-area.

Let the external heat flux with $q_{0}$ be led to ATE (see Figure 2). According to the tensor description of the Seebeck effect, the electric field intensity vector $E$ is

$$
\boldsymbol{E}=-\hat{e} \times \nabla T,
$$

where $\hat{e}(\mathrm{~V} / \mathrm{K})$ is a tensor of thermal EMF (Seebeck tensor) for an anisotropic medium; $\nabla T(\mathrm{~K} / \mathrm{m})$ is temperature gradient.

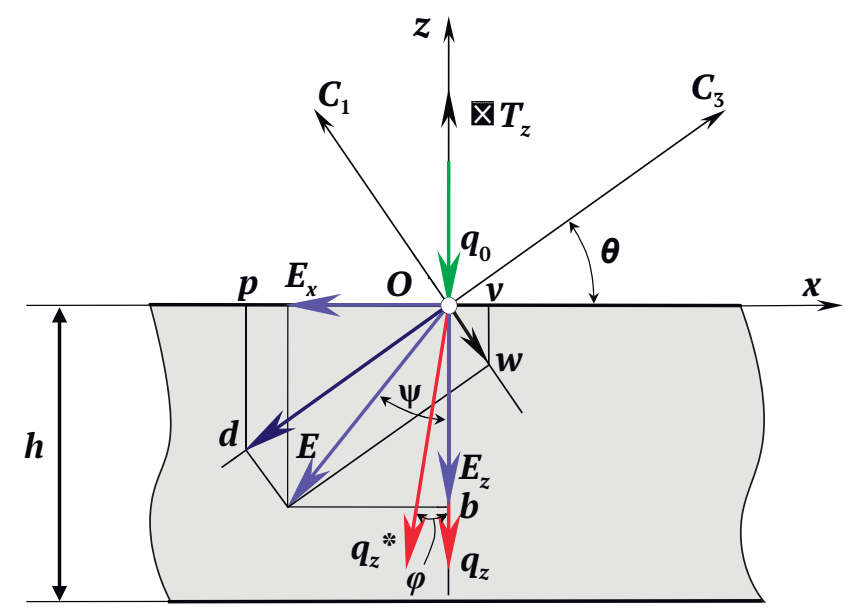

Figure 2. Heat flux and electric field intensity vectors in ATE directivity diagram: $O d$ and $O w$ are thermal EMF vector projections on crystallographic axes.

Since the heat flux vector in the ATE will deviate from the original direction (Figure 2), the temperature difference will occur not only in $z$-direction but also in $x$-direction. According to Fourier's law, the heat flux per unit area vector along the $z$-axis in the ATE is

$$
q_{z}=-k_{z} \frac{\nabla T_{z}}{a},
$$

where $k_{z}\left(\mathrm{~W} / \mathrm{m}^{2}\right)$ and $\nabla T_{z}(\mathrm{~K} / \mathrm{m})$ are ATE conductivity and temperature gradient in the $z$-axis direction, respectively.

Thermal EMF, according to [10], is

$$
E=\varepsilon_{x z} \frac{\partial T}{\partial z} l,
$$

where $\varepsilon_{x z}(\mathrm{~V})$ is the component of the secondary diagonal of the thermal EMF tensor in the $x y z$ coordinate system.

Due to that the thickness of ATE $a$ (see Figure 1) being small, temperature gradient in $z$-direction:

$$
\frac{\partial T}{\partial z}=\frac{\nabla T_{z}}{a}=\frac{T(a)-T(0)}{a} .
$$


Taking into account the continuity of the components of the external heat flux $q_{0}$, we get

$$
\left.q_{0 z}\right|_{z=0}=-\left.k_{z z} \frac{\partial T}{\partial z}\right|_{z=0}=-k_{z z} \frac{\nabla T_{z}}{a},
$$

where $k_{z z}(\mathrm{~W} / \mathrm{m})$ is the component of the conductivity tensor in the $x y z$ coordinate system.

Formula (4) takes the following form:

$$
E=\varepsilon_{x z} \frac{q_{0}}{k_{z z}} l
$$

and ATE sensitivity is

$$
s_{0}=\frac{E}{q_{0} A}=\frac{\varepsilon_{x z}}{k_{z z} b}
$$

Thus, the volt-watt sensitivity is determined by the components of thermal EMF and the conductivity tensor, and ATE width. The maximum volt-watt sensitivity $S_{0 \max }=S_{0}\left(\theta_{\text {opt }}\right)$ can be achieved by optimally deflecting the ATE trigonal plane by angle $\theta_{\text {opt }}$. The monograph [14] shows that:

$$
\theta_{o p t}= \pm \operatorname{arctg} \sqrt{\frac{k_{x x}}{k_{z z}}}
$$

The maximum sensitivity therefore also depends on the conductivity tensor components.

\section{GHFS Design}

The GHFS used in our experiments can be divided into two groups: GHFS based on single-crystalline bismuth with the purity of 0.9999 and HGHFS based on steel+nickel, copper-constantan and chromel-copel. There are other compositions, which are described in more detail in [14].

Figure 3 shows both types of GHFS. They differ in volt-watt sensitivity and operating temperature range. Bismuth-based GHFS has a sensitivity of $2 \ldots 10 \mathrm{mV} / \mathrm{W}$, while HGHFS sensitivity rarely exceeds $1 \mathrm{mV} / \mathrm{W}$. This is due to the fact that a bismuth-based GHFS is made in the battery form: the bismuth bricks are sequentially soldered with each other (Figure 3a), which multiplies the signal generated. A HGHFS is made up of alternating layers of different materials.

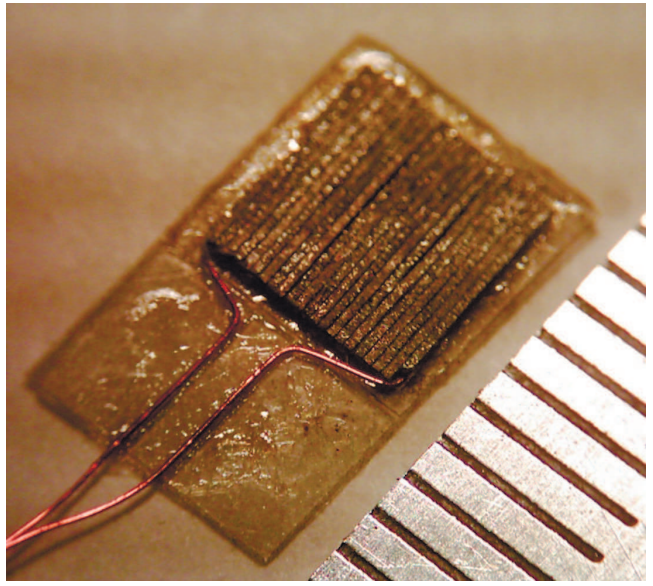

(a)

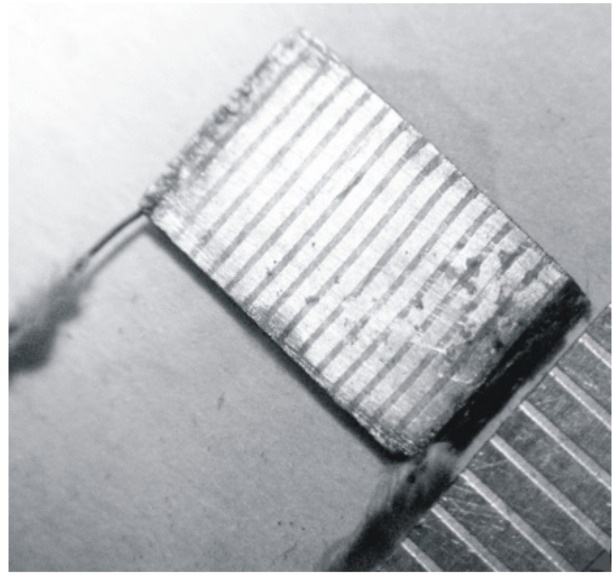

(b)

Figure 3. A bismuth-based gradient heat flux sensor (GHFS) (a) and a steel+nickel-based heterogeneous GHFS (HGHFS) (b). 
The operating temperature range of a bismuth-based GHFS is limited by its melting point ( $547 \mathrm{~K})$. The melting point of HGHFS can be significantly higher. For example, steel+nickel-based HGHFS works up to $1573 \mathrm{~K}$. It has been successfully applied in boiler furnace and diesel engine cylinder [14].

\section{GHFS Calibration and Measurement Uncertainty}

Each GHFS is calibrated on special benches and depends on the sensor type. Bismuth-based GHFS calibration was developed in the late 1990s. It is described in monograph [14]. This paper focuses on HGHFS calibration.

HGHFS should be calibrated in the working temperature range determined by the experiments' objectives. Peter the Great Saint Petersburg Polytechnic University, "NPO Etalon" and The D.I. Mendeleev All-Russian Institute for Metrology (VNIIM) jointly created the calibration bench shown in Figure 4 . The bench allows one to calibrate in the temperature range from 25 to $800{ }^{\circ} \mathrm{C}$.

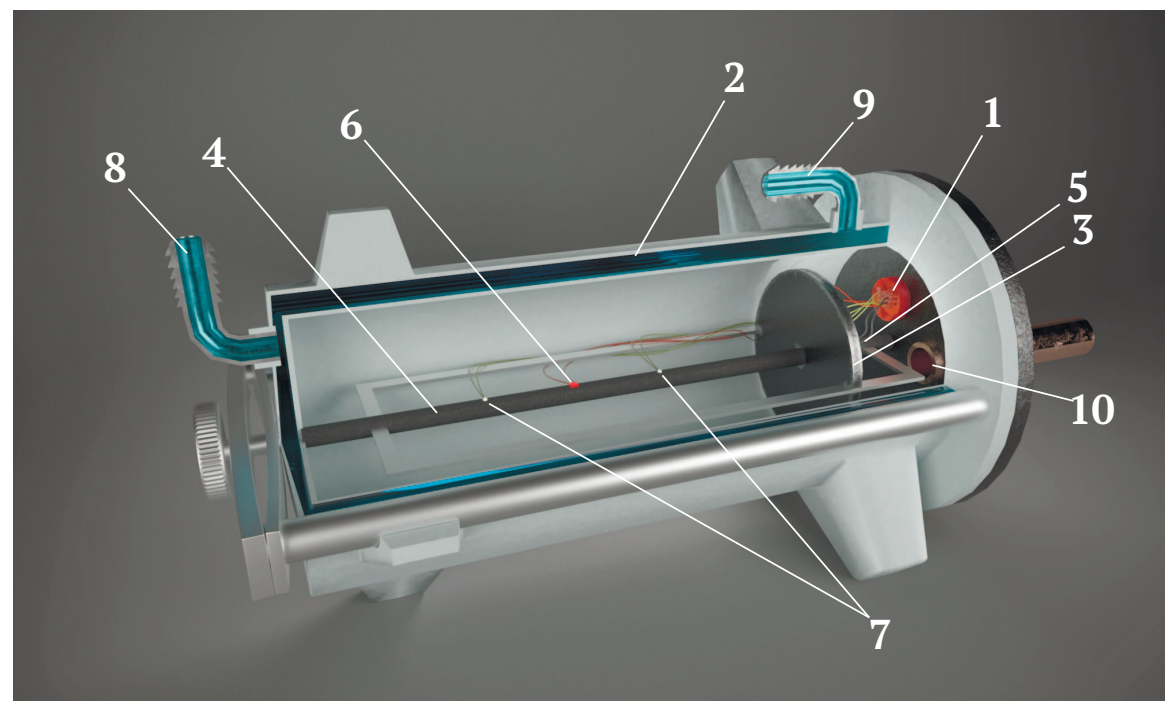

(a)

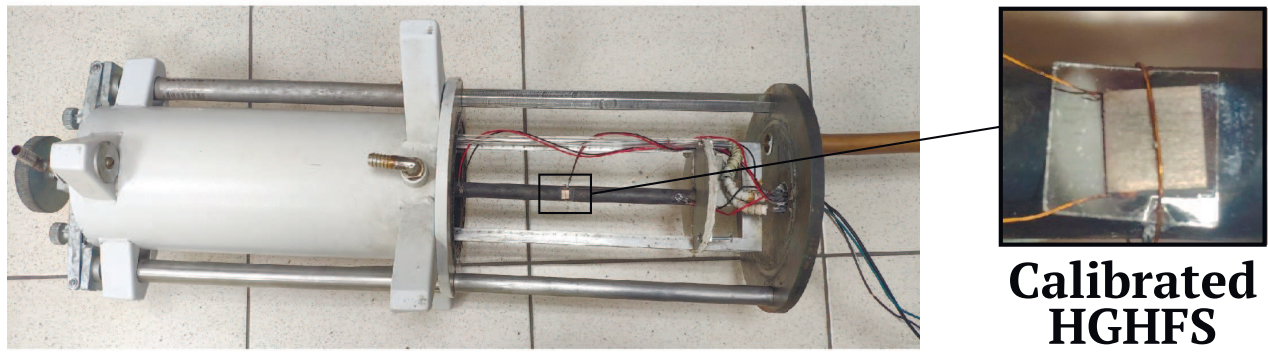

(b)

Figure 4. Calibration bench scheme (a) and appearance (b): 1-connector; 2-cylinder with coolant jacket; 3-tube holders; 4-stainless steel tube; 5-ohmic heater; 6-calibrated HGHFS; 7-thermocouples; 8, 9-water supply and discharge sockets, respectively; 10-vacuum socket.

The bench is a cylinder with a coolant jacket (2). The stainless steel tube (4) with a heater (5) inside is fixed on the cylinder axis in tube holders (3). The calibrated HGHFS (6) and thermocouples (7) are installed on the tube's (4) surface to control uniformity of tube heating. The cylinder (2) is cooled by the circulated water in the coolant jacket. Water is supplied and drained through sockets 8 and 9 . The cavity is vacuumed through socket 10 . 
In a sufficiently deep vacuum, heat is only transferred by heat conduction and radiation [15]. However, the heat conduction can be neglected, since:

$$
\frac{\sum q-q_{\text {cond }}}{\sum q}<1 \%
$$

where $q_{\text {cond }}\left(\mathrm{W} / \mathrm{m}^{2}\right)$ is heat flux due to heat conduction and $\sum q\left(\mathrm{~W} / \mathrm{m}^{2}\right)$ is total heat flux.

Our bench maintains a vacuum of $10^{-4} \mathrm{~mm}$ of mercury. It is achieved by using a system of fore pump VR005-21 and oil-vapor vacuum pump NDVM-100 (Figure 5).

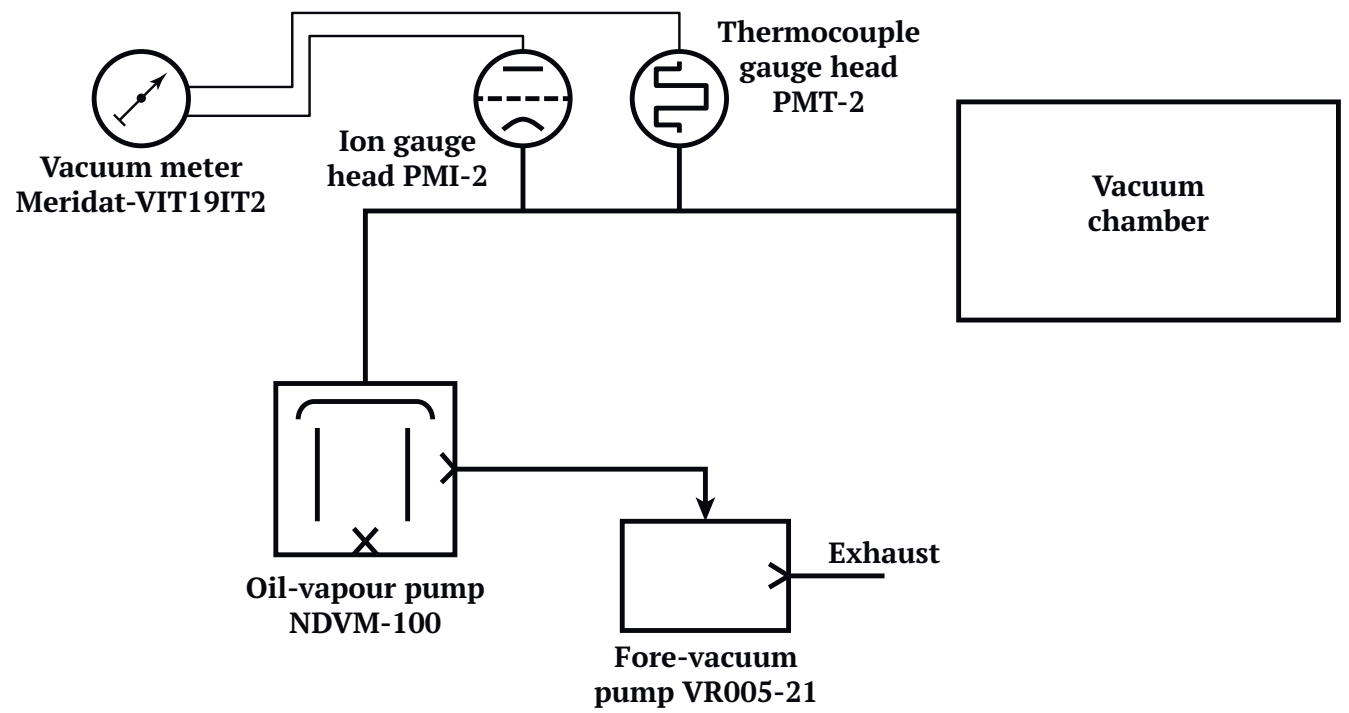

Figure 5. Exhaust cart scheme.

Bench heater 5 is powered by a GPR-73060D power supply. The power output of the heater is controlled by a voltmeter and ammeter. HGHFS and thermocouple signals are measured with the multimeter Fluke 289. The calibration is performed in close to steady-state. The temperature of the heater was controlled by changing the power. Heat flux was estimated as one Joule:

$$
P=I U(\mathrm{~W}),
$$

where $U(\mathrm{~V})$ is voltage; $I(\mathrm{~A})$ is heater circuit current.

Heat flux per unit area on tube 4's surface (see Figure 5):

$$
q=\frac{P}{\pi d l}=\frac{I U}{\pi d l}\left(\mathrm{~W} / \mathrm{m}^{2}\right),
$$

where $d=13 \mathrm{~mm}$ and $l=220 \mathrm{~mm}$ are tube 4 's diameter and length, respectively.

Volt-watt sensitivity of calibrated HGHFS:

$$
S_{0}=\frac{E \pi d l}{U I F}(\mathrm{mV} / \mathrm{W})
$$

As an example, Figure 6 shows the calibration results of the copper+nickel-based HGHFS. Dependence of volt-watt sensitivity on temperature can be described by the following equation:

$$
S_{0}=4.78 e^{-0.011 t}(\mathrm{mV} / \mathrm{W})
$$


where $t\left({ }^{\circ} \mathrm{C}\right)$ is GHFS temperature.

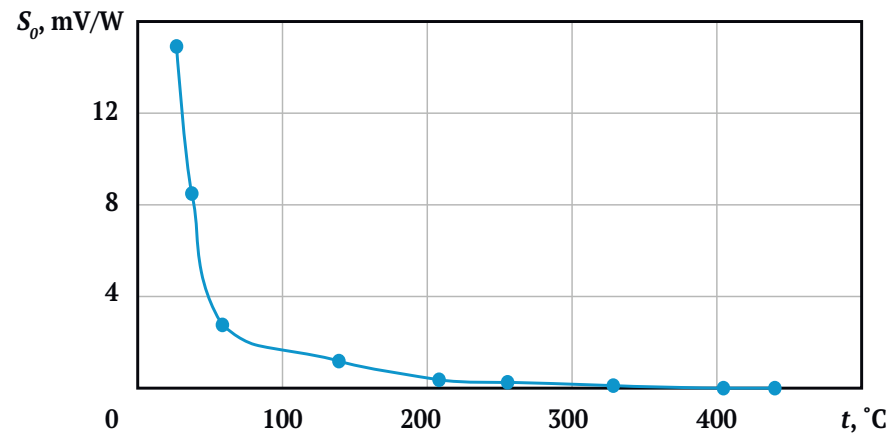

Figure 6. Results of copper+nickel HGHFS calibration.

A similar dependence is typical for all metal+metal HGHFS.

The relative expanded GHFS calibration uncertainty was calculated according to ISO/IEC GUIDE 98-4:2012—Uncertainty of Measurement [16]. It did not exceed 3.8\%.

\section{Application of GHFS}

In 1996-2020 there were several heat transfer studies in laboratory and industrial conditions using gradient heatmetry. GHFS and GHFS-based measuring devices were shown to be possible and reasonable to use for temperature determination, flow rate of liquid or gas, frictional shear stress, electric current power, etc. The main results are given in the monograph [14]. We will focus on recent studies of the Science Educational Centre "Energy Thermophysics" laboratories of Peter the Great Saint Petersburg Polytechnic University.

\subsection{Convective Heat Transfer}

Convective heat transfer was studied in an open-circuit subsonic wind tunnel (Figure 7).

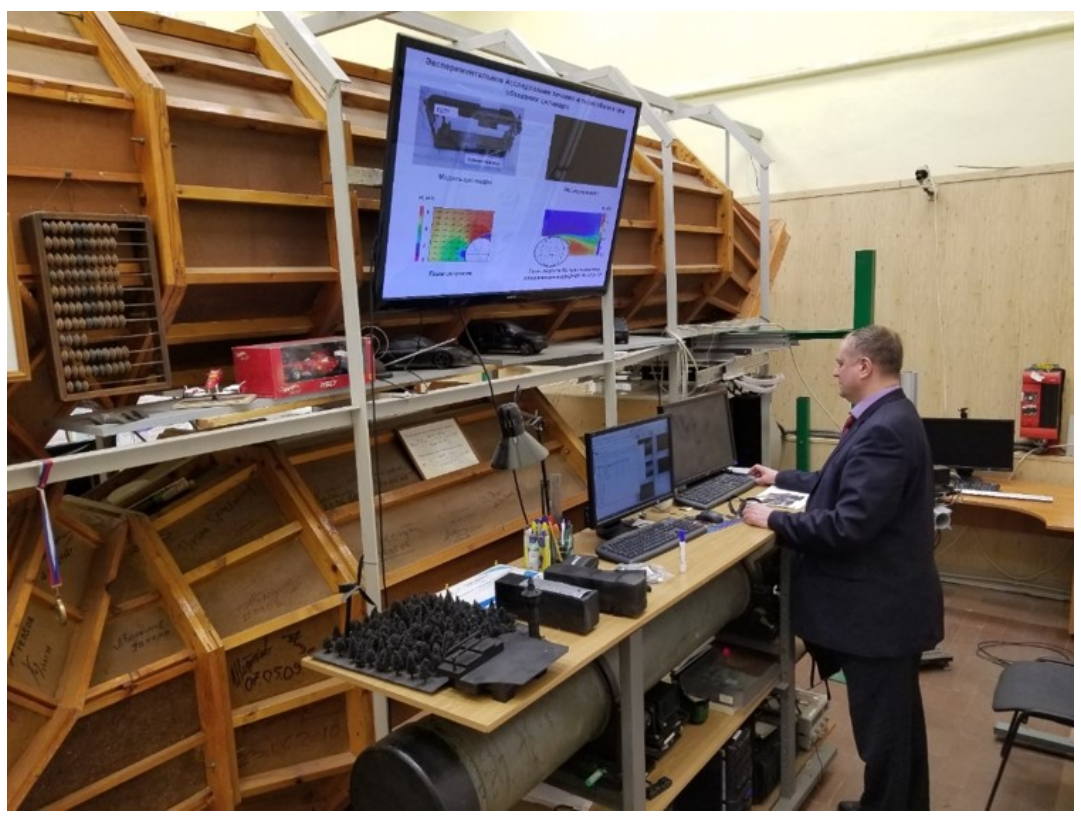

Figure 7. Wind tunnel. 
The tunnel is equipped with an air-water cooler, which is allowed to keep the flow temperature almost constant with a change of $0.1 \ldots 0.2^{\circ} \mathrm{C}$ during long experiments. The working area of the tunnel is equipped with an Eiffel chamber made of Plexiglas. The tunnel is also equipped with several devices, including a Schlieren optical system.

We have combined gradient heatmetry with PIV (Particle Image Velocimetry) and temperature measurement in a series of studies. We initially investigated one of the classic heat transfer tasks: cross-flow around a heated circular cylinder [17]. A $600 \mathrm{~mm}$ long and $66 \mathrm{~mm}$ in diameter hollow cylinder was used as the model. The cylinder was supplied with saturated steam at ambient pressure, which provided a constant cylinder surface temperature close to $100{ }^{\circ} \mathrm{C}$. A bismuth-based GHFS was installed on the cylinder surface, and the model could rotate around the axis. This design allowed us to measure local heat flux and heat transfer coefficient (HTC) over the cylinder's surface. In various experiments, the free-stream velocity $W$ changed (Reynolds number $R e=\frac{W d}{v}$, where $d$ is cylinder diameter and $v$ is kinematic air viscosity). Velocity field near the cylinder was visualized using PIV by POLIS synchronously [18] with the heatmetry. Detailed experimental descriptions are summarized in [14]. Note that the Eiffel chamber allows long experiments without tracer dispersion, which greatly simplifies the work with PIV.

The heat transfer augmentation near a circular single cylinder was investigated by gradient heatmetry. To shift the point of laminar-turbulent transition [19], thin rods-turbulizers were used, which were installed along with the element of the cylinder (Figure 8 ). The turbulizers were symmetrically spread in relation to the frontal element (stagnation point) at an angle $\psi$, had a diameter $d_{T}$ and were installed close to the cylinder and with a gap $\delta$. The values $d_{T}, \delta$ and $\psi$ were optimized (according to heat transfer) during the experiment. The experimental results for $\operatorname{Re}=4.8 \times 10^{3}$ are shown in Figure 8 . Nusselt number $N u=\frac{h d}{k}$ was calculated by cylinder diameter $d$ ( $h$ is local HTC, $k$ is air conductivity).
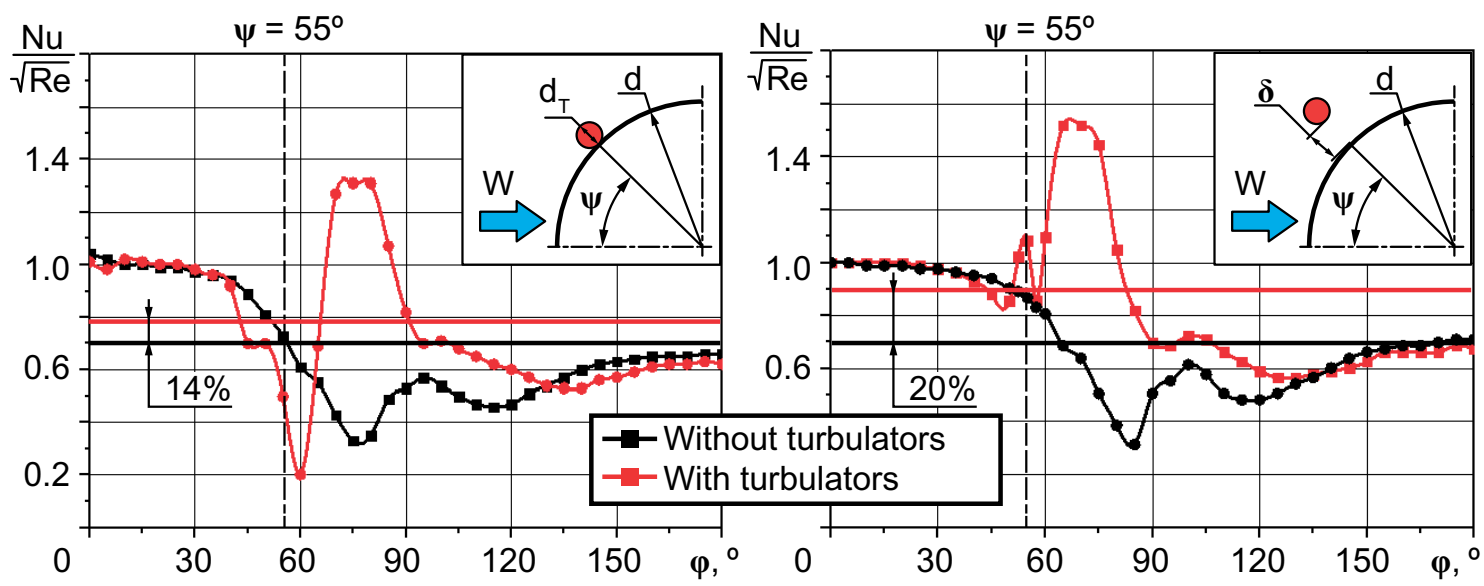

Figure 8. Heat transfer augmentation with turbulizers: turbulizers set without a gap, turbulizers set with a $\operatorname{gap}\left(d_{T}=1 \mathrm{~mm}, \delta=1.2 \mathrm{~mm}\right.$ at $\left.\operatorname{Re}=4.8 \times 10^{3}\right)$.

Installation of optimal shape turbulizers allowed us to increase local HTC by $45 \%$, and the average HTC by $20 \%$ (red line) compared to the cylinder without turbulizers (black line).

PIV with gradient heatmetry made it possible to visualize the flow near the hot model's surface (Figure 9).

Figure 9 shows the shift of the separation point. The vortex street expansion angle is smaller for a cylinder with turbulizers than for a smooth cylinder. Experiments on this topic are still ongoing. 


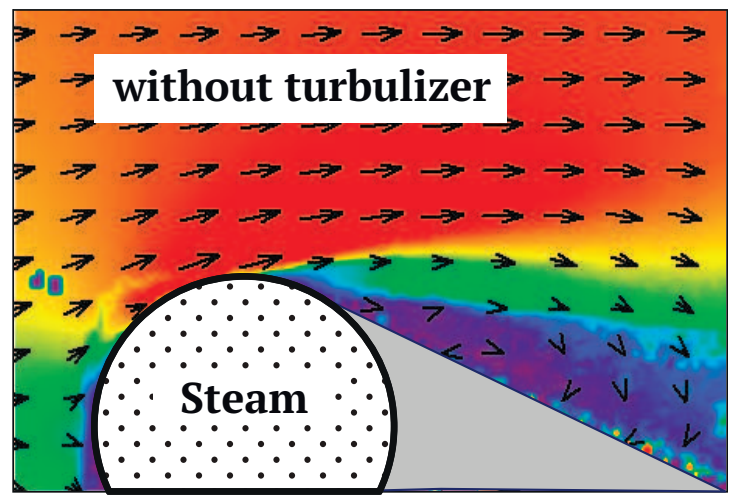

(a)

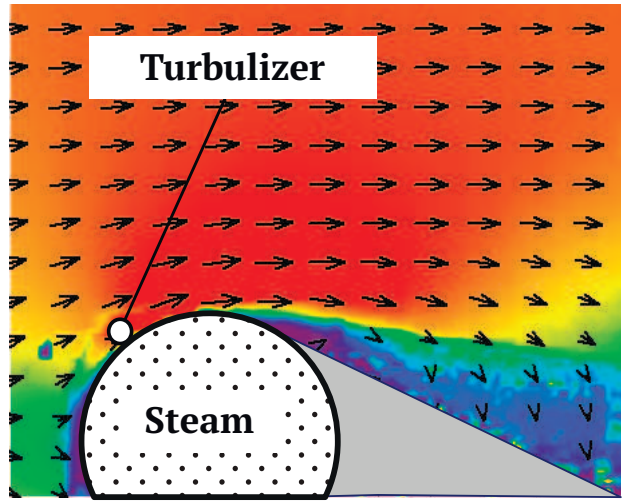

(b)

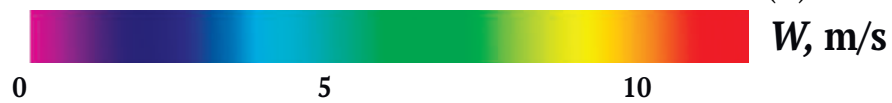

Figure 9. Averaged velocity fields during flow around a smooth cylinder (a) and a turbulizers-equipped cylinder (b).

Experiments were done on the model of two cylinders spread by distance $S$ (Figure 10). Both cylinders were heated with saturated steam, and the rear cylinder was rotated.

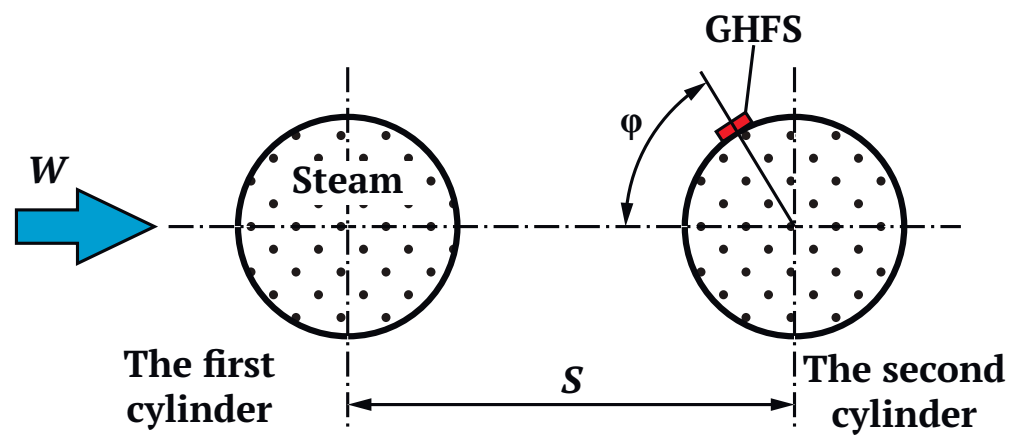

Figure 10. Experience with two cylinders.

The study was done in the range of Reynolds numbers $R e=(0.48 \ldots 48) \times 10^{3}$. Figure 11 shows the instantaneous velocity fields between the cylinders for different regimes. Vectors show the flow direction and colors show velocity values. The gray area is where the laser could not light the tracers.

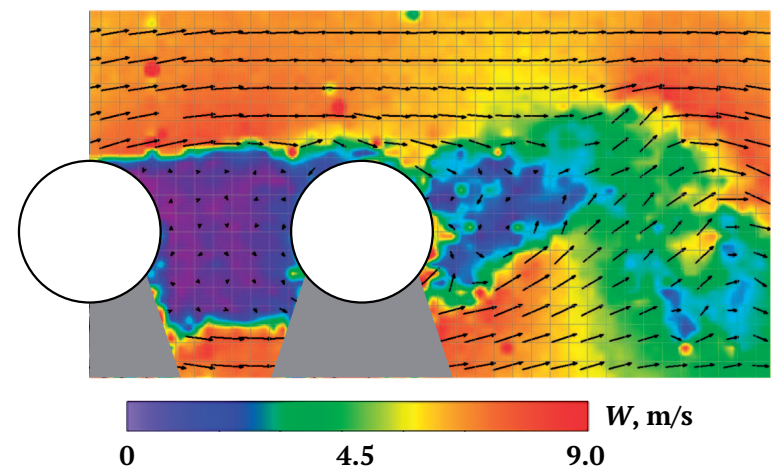

(a)

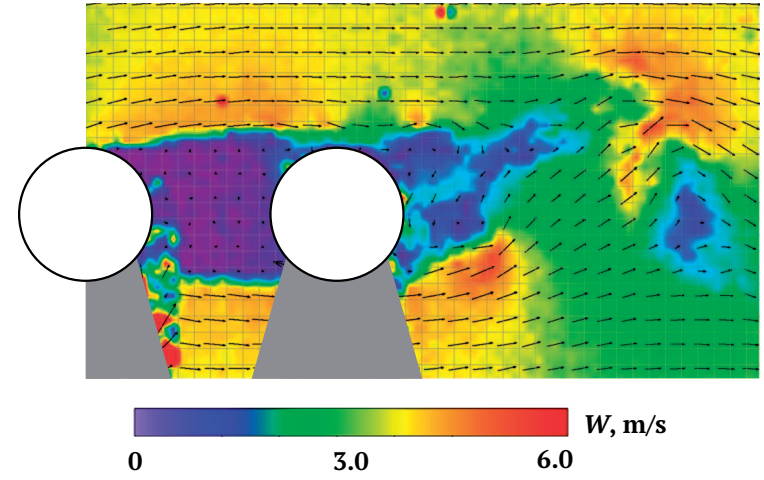

(b)

Figure 11. Instantaneous velocity fields, $S=d$ : (a) $-R e=9600 ;(\mathbf{b})-R e=4800$. 
The stagnation zone between the cylinders, where the air velocity is minimal, was detected. The velocity in the wake is greater than that of the incoming flow. As the distance $S$ increases, the vortex street expansion angle increases.

Using GHFS, we have obtained the HTC distribution on the surface of the second cylinder, depending on the regime (Figure 12).

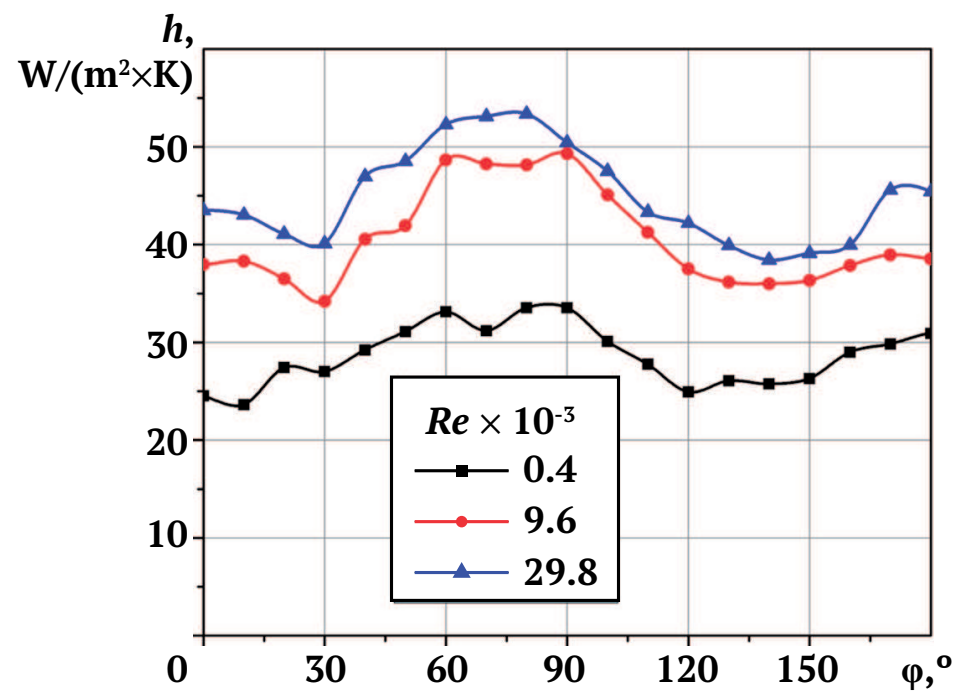

Figure 12. Local heat transfer coefficient distribution over the second cylinder's surface at $S=d$.

HTC distribution by the angle $\varphi$ of the second cylinder is different from the typical one of a single cylinder obtained by us and other authors [20]. Maximum HTC is in the area $\varphi=60 \ldots 90^{\circ}$, not at the front point. As the air velocity increases, HTC is expected to increase.

Unique GHFS response time allows us to estimate not only average level, but also fluctuations of heat flux and HTC. The local heat flux dispersion $D$ was calculated (Figure 13).

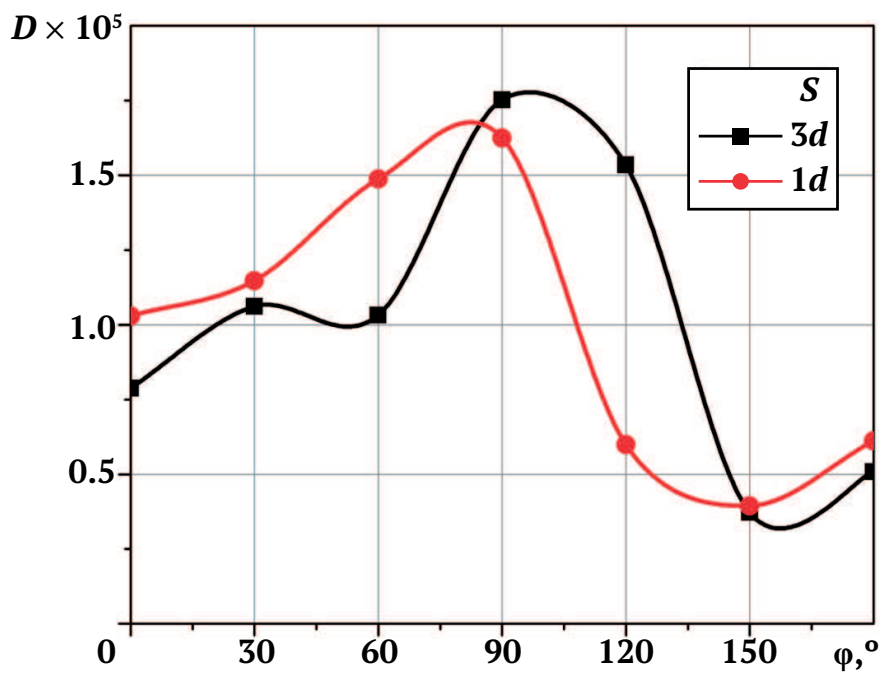

Figure 13. Heat flux dispersion over the second cylinder's surface. 
The maximum fluctuation for the second cylinder is also close to the angle $\varphi=90^{\circ}$, while for a single cylinder it is shifted to the stern area. The fluctuation level for the second cylinder is 10 times less than for a single cylinder, so the first cylinder stabilizes the flow and HTC.

A model based on the hollow cylinder described above was created to study the heat transfer at circular fins and finned tubes. A hollow circular fin was mounted on it, which allowed us to simulate an "ideal" fin with infinite conductivity. In other experiments, a VT-22 titanium fin was mounted on the cylinder (Figure 14).

To calculate HTC, we supplemented PIV and gradient heatmetry with a third method, i.e., the temperature measurement. The single-fin experiment diagram is shown in Figure 14. The solid fin was investigated in the same way.

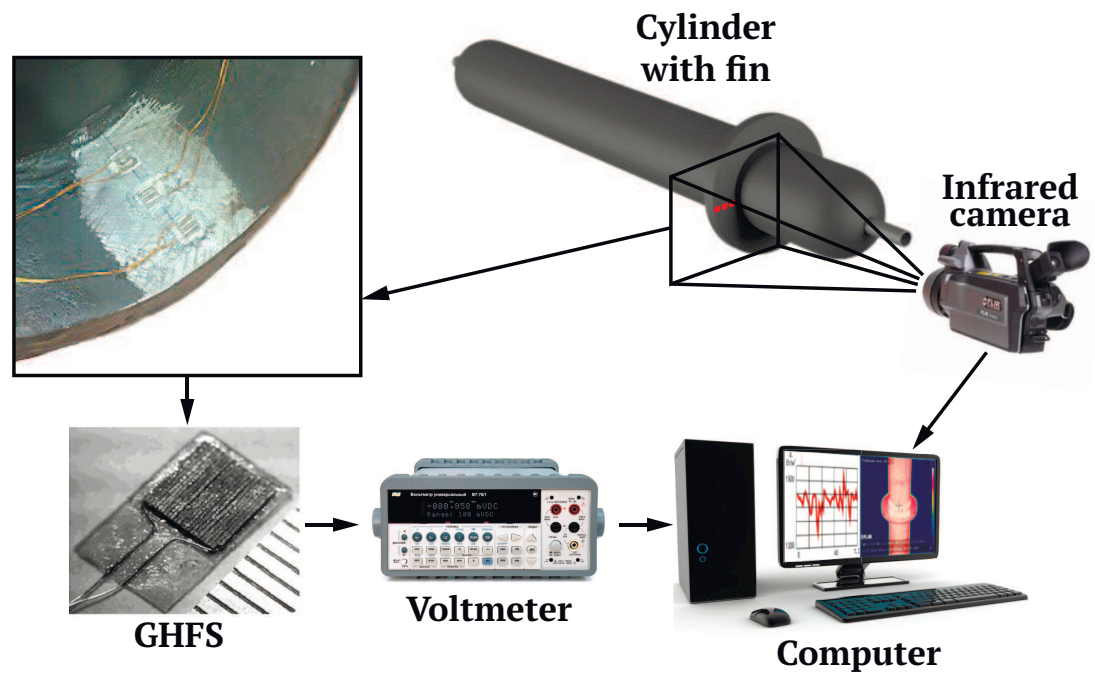

Figure 14. Scheme of the heat transfer experiment using a GHFS and a FLIR P640 infrared camera.

The FLIR P640 infrared camera was used to measure the single fin temperature. Figure 15 shows the temperature fields on the isothermal and non-isothermal fins.

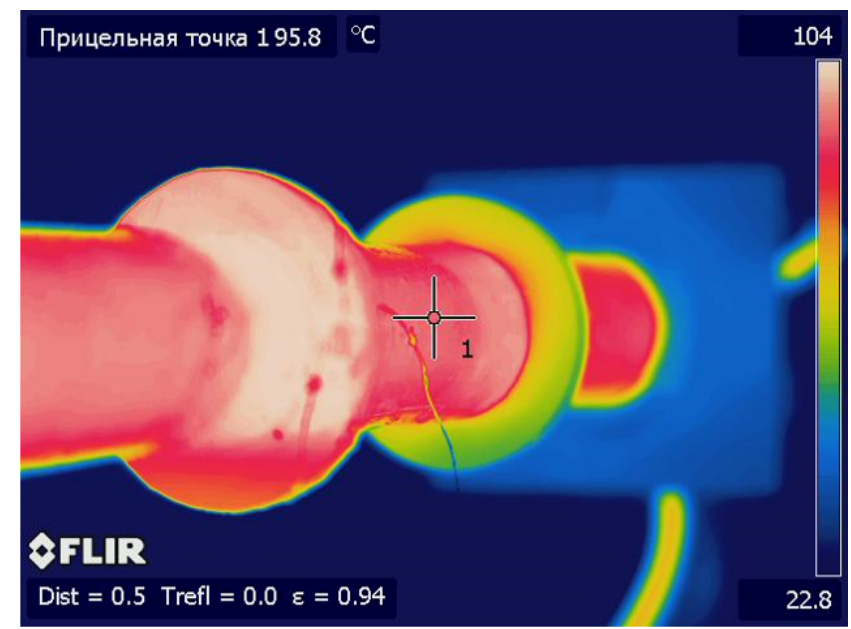

Figure 15. Image taken with an infrared camera on a model with hollow (front) and solid (rear) fins. 
Figure 16 shows that HTC distribution both on the "ideal" fin and on a VT-22 titanium fin is significantly uneven.

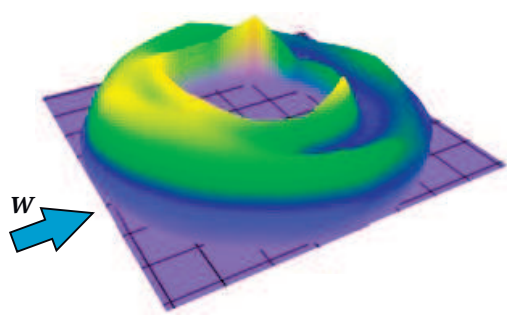

(a)

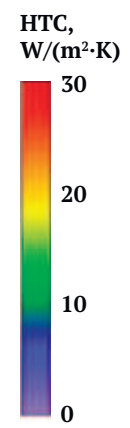

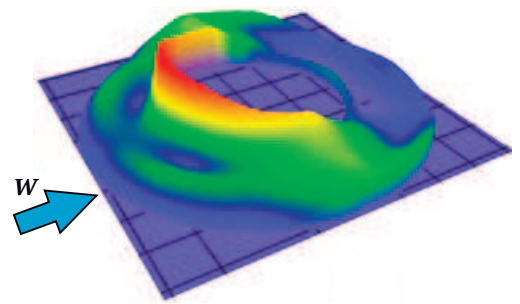

(b)

Figure 16. Local heat transfer coefficient distribution: on the hollow fin (a); on the VT-22 titanium fin (b).

The flow visualization near a single hollow circular fin is shown in Figure 17. The pictures are averaged with respect to 1000 frames and correspond to the flow pattern described in [21]. Due to the low height of the fin and a sharp edge, the pictures show a flow breakdown and its further adjoining with a small swirl, which, however, does not have time to form a full vortex above the fin. The flow structure typical for the flow of a smooth round cylinder can be seen at enough distance from the fin (around the caliber).
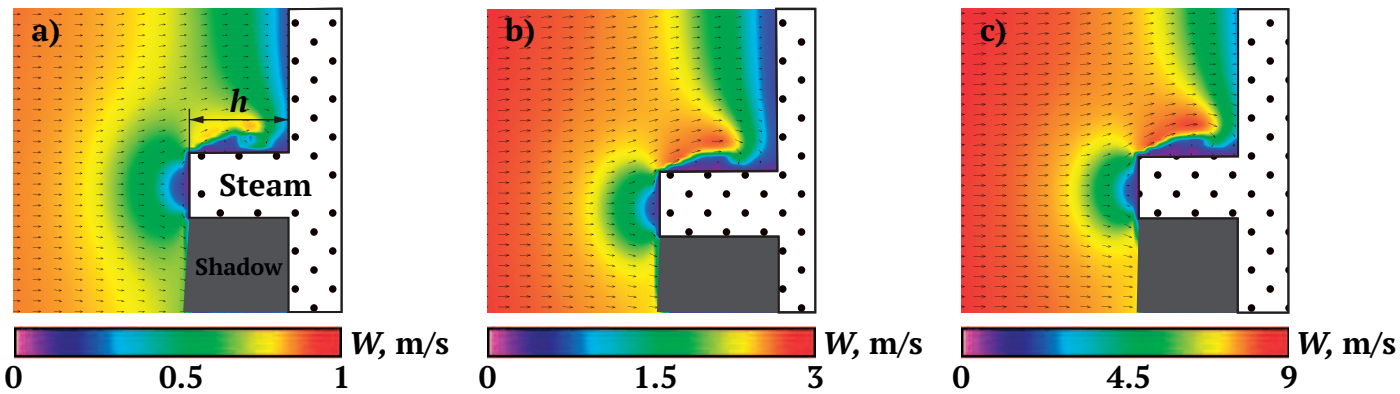

Figure 17. Velocity field near single fin height $h=20 \mathrm{~mm}$ : (a) $R e=0.9 \times 10^{4}$; (b) $R e=2.1 \times 10^{4}$; (c) $R e=4.2 \times 10^{4}$.

We investigated the finned tube in the same range of Reynolds numbers. The flow between the fins was visualized when three fins were mounted on the cylinder. The results for different fin spacings are shown in Figure 18.

It can be seen that the vortex above the first fin is typical for single fin flow. If the distance between the fins is smaller, the flow in the interspace becomes asymmetrical and it also affects the local HTC.

Gradient heatmetry, combined with PIV and thermal imaging, made it possible to study the flow and heat transfer on the surfaces of smooth circular cylinders, a cylinder with turbulizers and the circular fin and finned tube. The experiment succeeded in obtaining real-time data on the matching of flow and heat transfer near different heat transfer surfaces. 

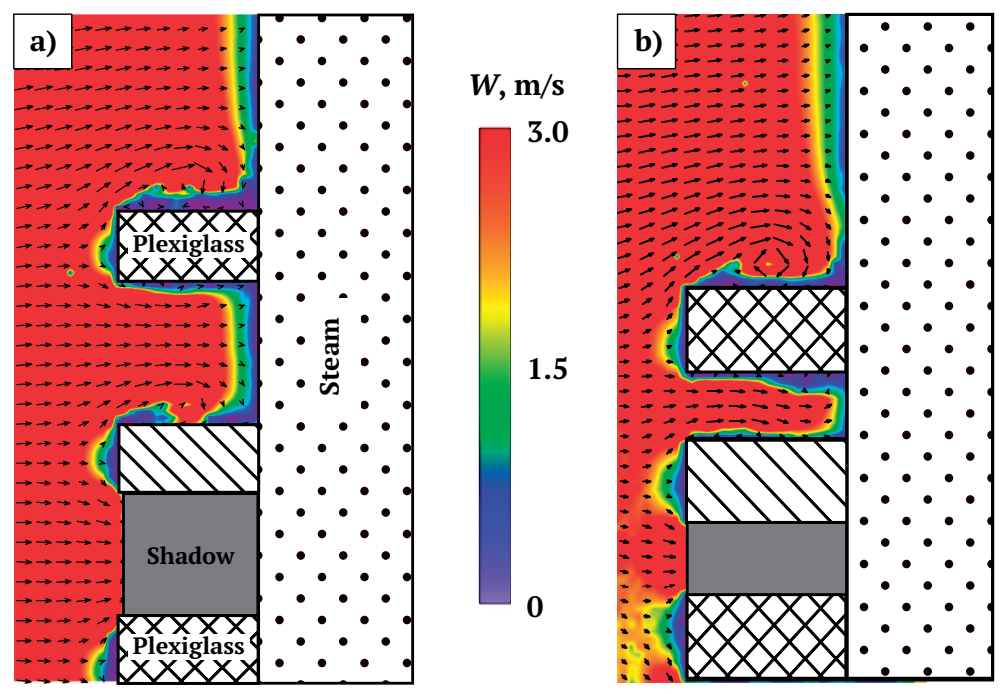

Figure 18. Velocity field near finned cylinder at $R e=1.3 \times 10^{4}$ with fin spacing: (a) $u=20 \mathrm{~mm}$; (b) $u=10 \mathrm{~mm}$.

\subsection{Heat Transfer during Steam Condensation}

Nowadays temperature measurement is the most used method for experimental research in this field. The surface temperature is measured by thermocouples and resistance thermometers. The heat flux and HTC are determined by calculation [22,23]. This approach is methodically not strict. Heat flux fluctuations [24] cannot be measured correctly by sufficiently inertial thermocouples. We have started to apply GHFS in the study of condensation relatively recently but managed to obtain results confirming the applicability of gradient heatmetry. Our heat transfer studies during condensation of saturated steam go in two directions: condensation on the outer surfaces and on the inner surfaces of the tubes.

\subsubsection{Condensation on the Outer Surfaces of The Tubes}

In the setup, five bismuth-based GHFS with dimensions of $2.3 \times 10.5 \times 0.3 \mathrm{~mm}$ installed flush with the tube surface. Determining the HTC requires knowing the wall temperature at the GHFS installation site. Junctions of semi-artificial thermocouples were placed on one element with GHFS. Hot junctions were the contacts between the copper wires and the tube's surface, while cold junctions were the contacts between the copper wire and the tube material (steel) at ambient temperature.

The experimental section consisted of two coaxial tubes. The stainless-steel $1.25 \mathrm{~m}$ long tube with diameter $d=0.02 \mathrm{~m}$ and wall thickness $\delta=2 \mathrm{~mm}$ was surrounded by an external $1 \mathrm{~m}$ long tube (casing) made of reinforced rubber hose $(D=65 \mathrm{~mm}, \delta=5 \mathrm{~mm})$. The inner tube was fixed in the casing by the two rubber stoppers; GHFS and thermocouple wires were let out through the upper stopper (Figure 19a). When studying film condensation, the tube's surface should be free of protruding parts and wires [25]. For this purpose, two rods with a diameter of $3 \mathrm{~mm}$ were mounted $9 \mathrm{~mm}$ from the test section, along which the wires were tapped (Figure 19b). 


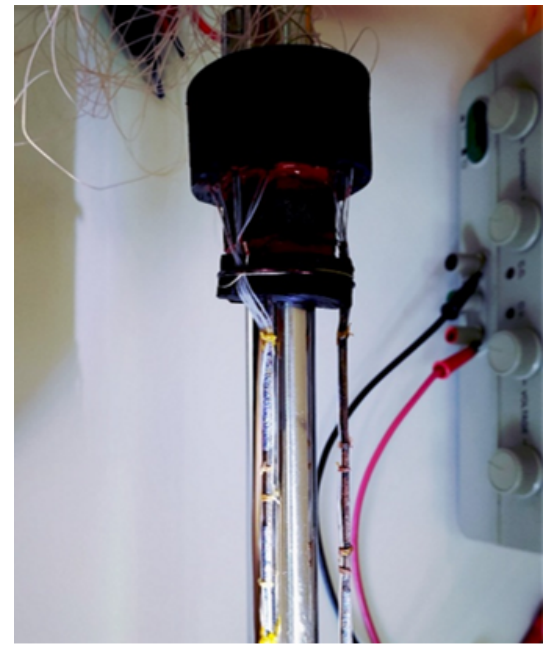

(a)

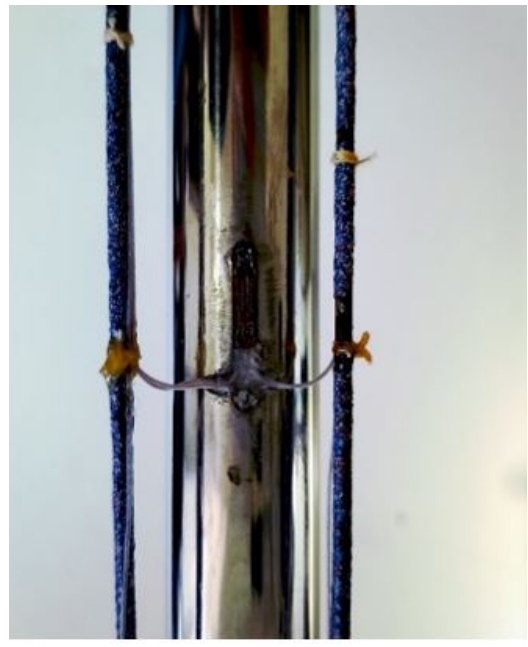

(b)

Figure 19. GHFS and thermocouple wire termination (a) and lead (b).

Saturated steam with a temperature close to $100^{\circ} \mathrm{C}$ and a mass flow rate of $10 \mathrm{~kg} / \mathrm{h}$ was fed into the space between the tubes from above, while cooling water with a temperature of $22{ }^{\circ} \mathrm{C}$ and a mass flow rate of $720 \mathrm{~kg} / \mathrm{h}$ was fed into the tube from below. The experimental section was equipped with devices for inclination $\left(\psi=0 \ldots 90^{\circ}\right)$ and rotation of the tube around its axis $\left(\varphi=0 \ldots 180^{\circ}\right)$, which allowed us to study the heat transfer at vertical, horizontal and inclined tube positions.

A feature of the bench was a measuring device based on a light-beam oscillograph to record GHFS and thermocouple signals. The light sources were replaced by laser modules whose beams were directed at galvanometer mirrors. The reflected beams were projected onto the remote screen. This method made it possible to record GHFS signals without the electrical interference that is unavoidable when using digital converters.

Figure 20 shows the time dependency of the heat flux (heat flux graph) obtained by condensing steam in a vertical tube. The signals of three GHFS are presented at $x=300 \mathrm{~mm}, 700 \mathrm{~mm}$ and $800 \mathrm{~mm}$, where $x$ is the distance from the upper cut of the tube.

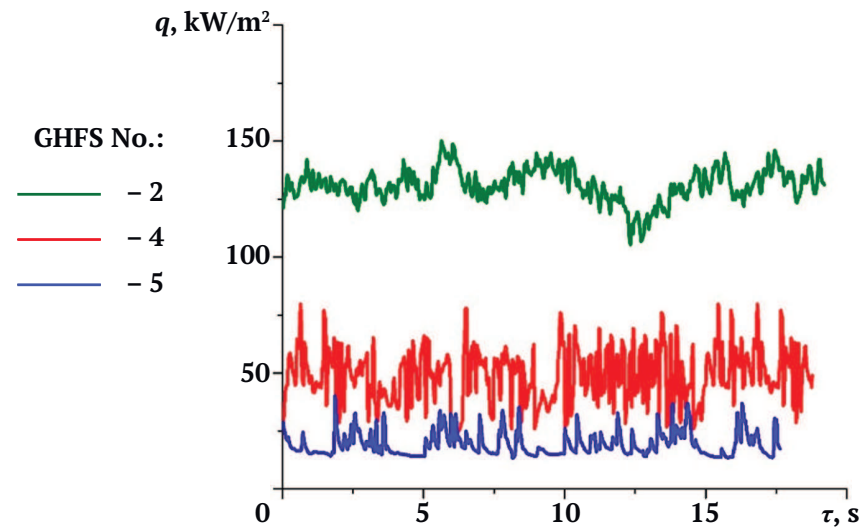

Figure 20. Heat flux graphs for vertical tube: $2-x=300 \mathrm{~mm} ; 4-x=700 \mathrm{~mm} ; 5-x=800 \mathrm{~mm}$.

The length-averaged heat flux on the tube was $133.4 \mathrm{~kW} / \mathrm{m}^{2}$ and yjr length-averaged HTC was $6.06 \mathrm{~kW} /\left(\mathrm{m}^{2} \times \mathrm{K}\right)$. That is close to the value calculated by the Nusselt formula, equal to $6.1 \mathrm{~kW} /\left(\mathrm{m}^{2} \times \mathrm{K}\right)$. The average HTC in the horizontal tube experiment was $5.54 \mathrm{~kW} /\left(\mathrm{m}^{2} \times \mathrm{K}\right)$. 
When examining the heat transfer on a tilted tube, its deviation from the vertical was changed within the limits of the tilt angle $\psi=0 \ldots 90^{\circ}$. The dependencies of dimensionless heat flux on the azimuthal angle $\varphi=180^{\circ}$ were constructed for three sites. Figure 21 shows the results of the experiments for $\psi=30^{\circ}$ and $\psi=80^{\circ}$.

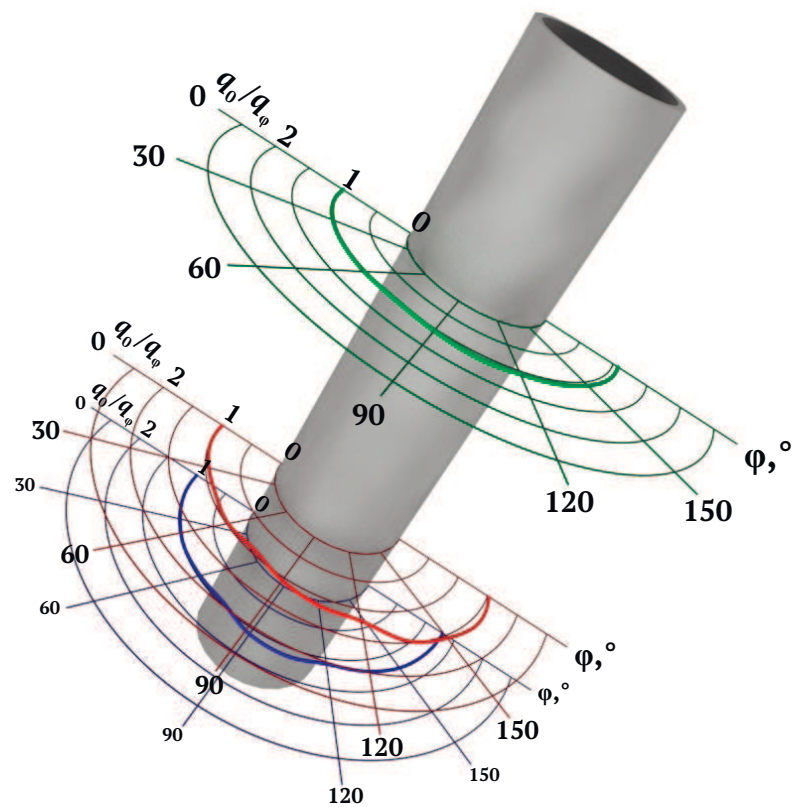

(a)

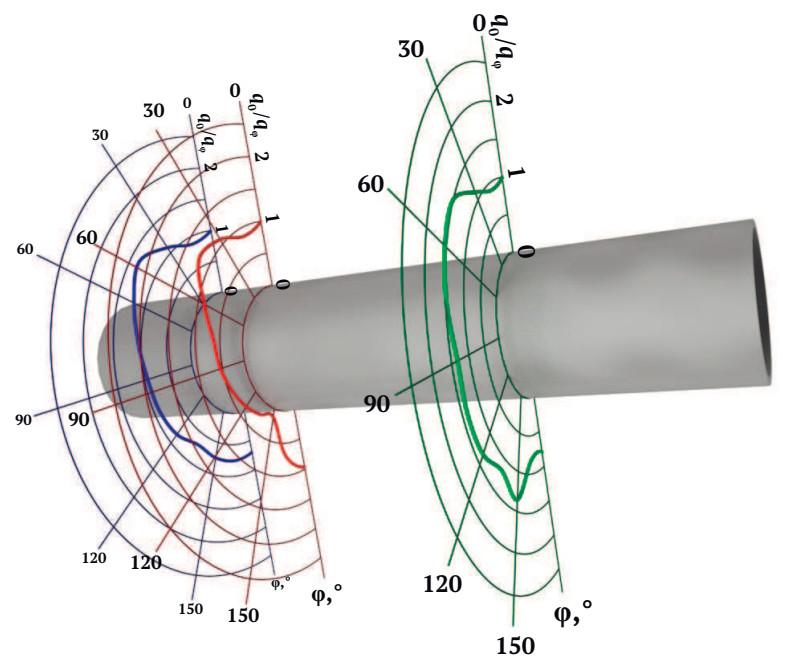

(b)

Figure 21. Relative heat flux: $2-x=300 \mathrm{~mm} ; 4-x=700 \mathrm{~mm} ; 5-x=800 \mathrm{~mm} ;(\mathbf{a})-\psi=30^{\circ},(\mathbf{b})-\psi=80^{\circ}$.

GHFS readings suggest that there is a bottom zone, the nature of which depends on the tilt angle. The optimal tube tilt in terms of heat transfer during condensation $\left(\psi=20^{\circ}\right)$ was determined under experimental conditions: on this slope, the heat flux is $12 \%$ higher, and HTC is $14.9 \%$ higher compared to the vertical tube. 


\subsubsection{Condensation on the Inner Surfaces of Tubes}

The $1 \mathrm{~m}$ long vertical steel tube had an inner diameter of $20 \mathrm{~mm}$ and a wall thickness of $0.5 \mathrm{~mm}$; it was surrounded by a coolant jacket. Segments with HGHFS (steel 12H18N10T+nickel) with holes for sensor wires and thermocouple electrodes were cut out in the tube using an electric discharge sawing machine (Figure 22). HGHFS were installed on a $0.05 \mathrm{~mm}$ thick mica sheet insulating substrate and then flush-mounted to the inner surface of the tube using epoxy resin. The thermocouples were placed close to the HGHFS. After mounting the HGHFS and the thermocouple, the segment was soldered back into the tube. Due to the low cutting thickness of the electric discharge sawing machine, no ledge was formed on the inner surface and no distortion of the condensate film was observed.

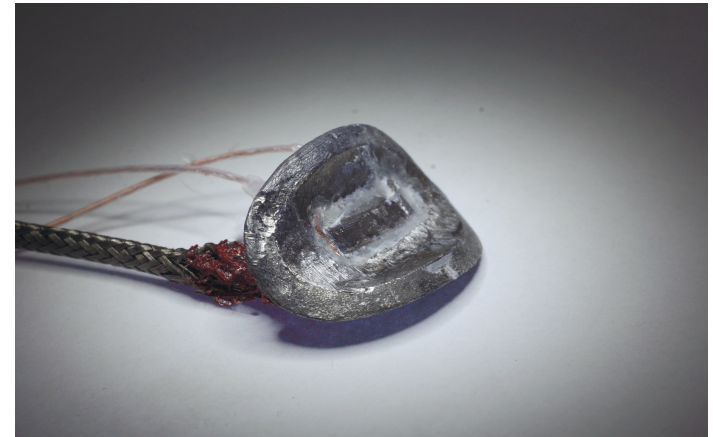

(a)

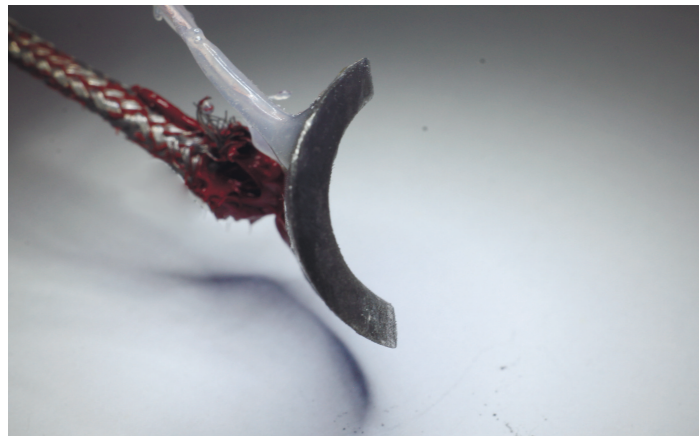

(b)

Figure 22. Measuring segment with mounted HGHFS: (a) — isometry and (b) — side view.

The segments were mounted with different pitches over the height of the tube. In the course of research, the steam generator power and the direction of water and steam supply were varied. In particular, the poorly studied condensation of steam in the tube during steam and condensate counter-flow was investigated.

Having performed a number of experiments, we confirmed the HGHFS' working ability under conditions of saturated steam condensation inside the tube [14]. The HGHFS' response time made it possible to detect heat flux fluctuations during steam condensation. Figure 23 shows the power spectral density (PSD) as a function of cooling water and steam mass flow rate. The heat flux fluctuation nature corresponds to the existing assumptions about the wave formation of the condensate film [26,27].

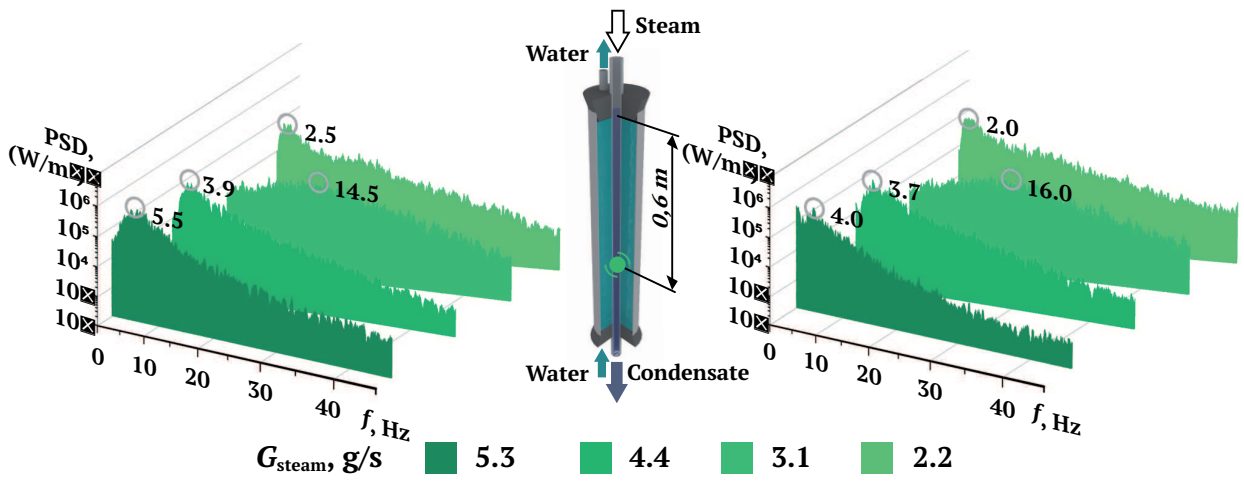

Figure 23. Heat flux fluctuation.

The experimental bench was then upgraded. The control section was attached to a rotating device with a separating disc, which allowed the tube to be tilted at an angle $\psi$ from the horizontal. Since the 
distribution of heat flux around the tube perimeter was asymmetrical, the tube with a disc was mounted on another rotating device that allowed the tube to be rotated around the axis at an angle $\varphi$ (Figure 24). This allowed only one sensor and one thermocouple to be used to measure heat flux and temperature along the inner tube perimeter.

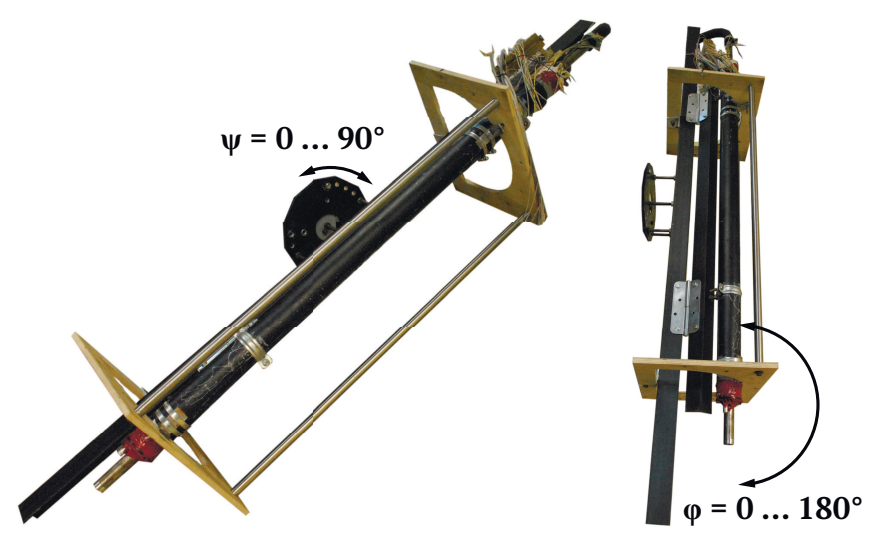

Figure 24. Experimental section with rotating mechanisms.

The results shown in Figure 25 were obtained at steam mass flow rate $G_{s}=4.4 \mathrm{~g} / \mathrm{s}$ for tilt angles $\psi$ from 0 to $60^{\circ}$. National Instruments' measurement and computing system (MCS) recorded HGHFS' and thermocouples' signals and then calculated local HTC. The results were averaged by normalizing the obtained by HTC in the upper cross-section of the tube.
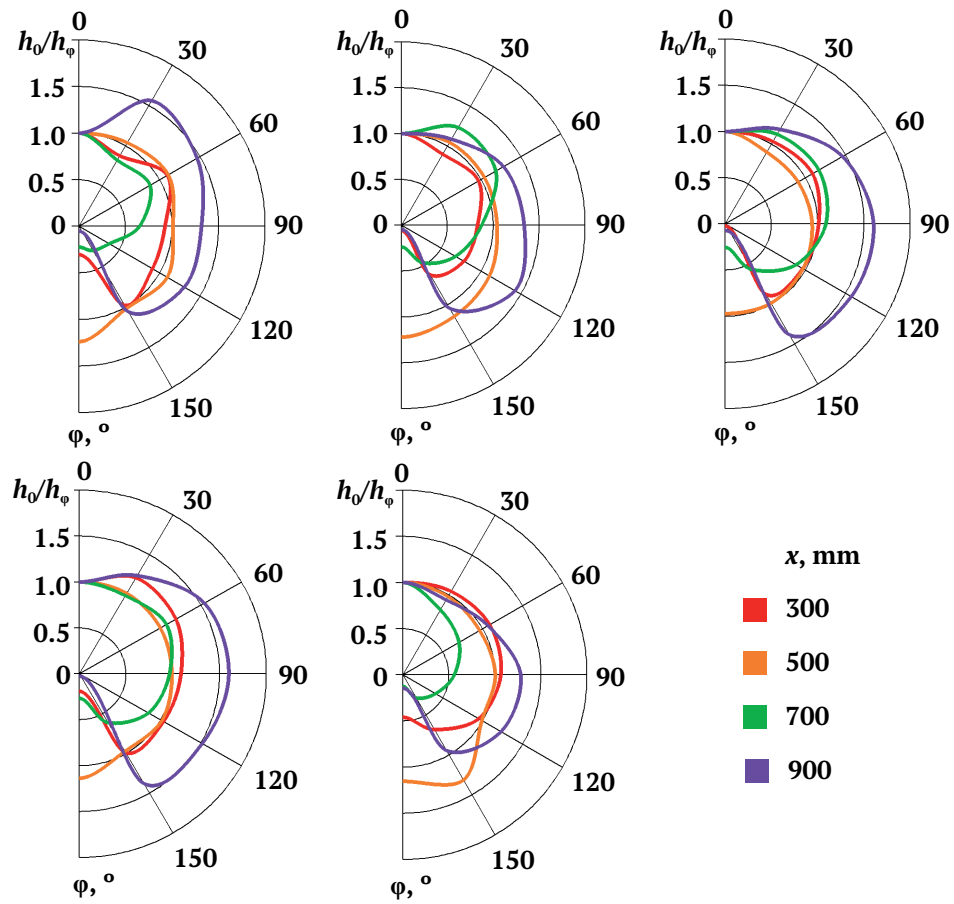

Figure 25. Relative local heat transfer coefficient around the tube perimeter; $x$ is distance from the upper section of the measuring section.

The graphs show a HTC decrease at the bottom of the tube due to the condensate film thickening. 


\subsection{Heat Transfer during Boiling}

One of the most under-explored problems in this field is non-isothermal surface boiling [28]. The application of HGHFS opens up new possibilities for researchers.

The first experiments were done on the sphere's surface, as this model excludes the influence of end effects. The sphere was made of titanium VT-22 (Figure 26).

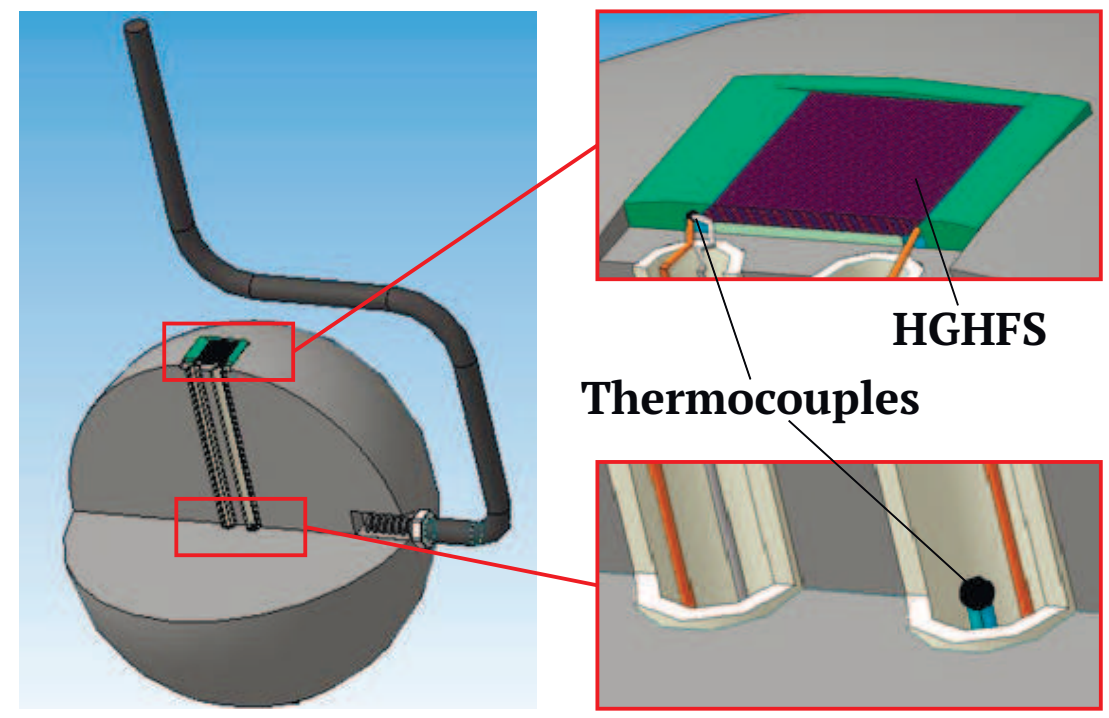

Figure 26. Model design.

A sampling was done to mount the sensor on the sphere surface. Copper+nickel-based HGHFS of $3 \times 3 \times 0.3 \mathrm{~mm}$ was installed flush with the surface and fixed with a special compound. Through-holes were made in the ball for the wires' and copper-iron thermocouples' installation (Figure 26).

The wires were fixed to the sensor by spot welding. The model after heat treatment and grinding is shown in Figure 27.

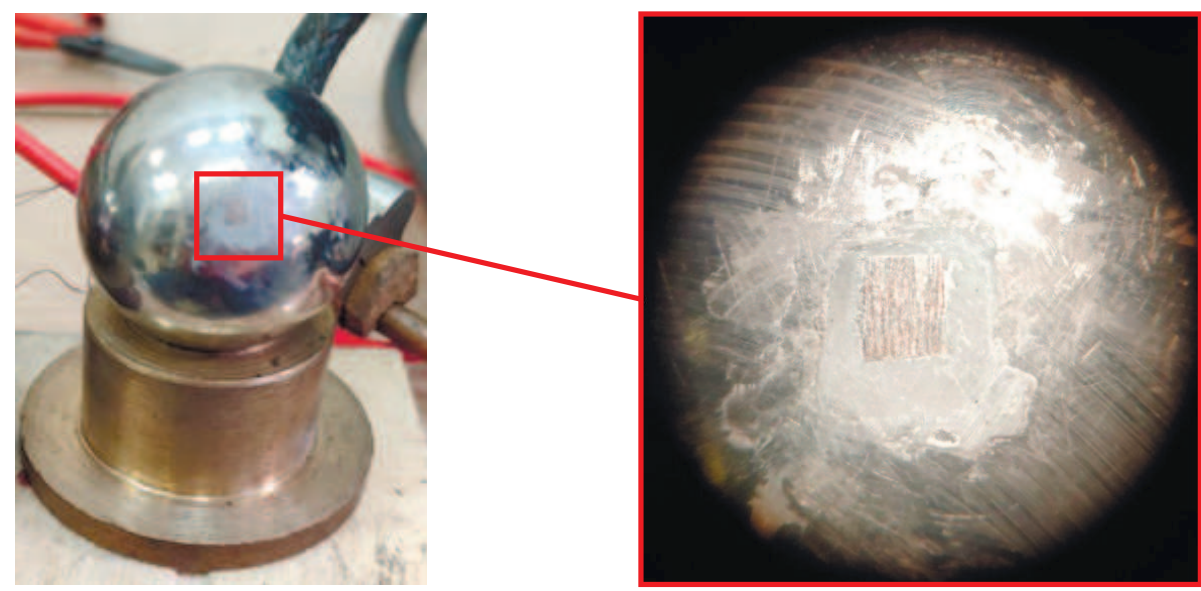

Figure 27. Experimental model with HGHFS.

The bench scheme is shown in Figure 28. Experimental model 1 was placed in the tube through furnace 2 and fixed in the furnace using the holder 3 . The temperature and uniformity of the sample heating were controlled by two thermocouples installed in the model, the readings from the MCS (8). 
When sample reach the desired temperature, the holder (3) was released and sample 1 was poured through the furnace into the water tank (4); thermocouples and HGHFS signals were recorded by the MCS (8) and boiling was simultaneously recorded by the high-speed camera "Evercam-4- $\mathrm{C}$ " (6). The temperature in the tank was controlled by a Fluke 289 with a K-type thermocouple (5), and the required water temperature level was maintained by an electric heater. Video footage was captured at $1000 \mathrm{fps}$, and data from the HGHFS and thermocouple were recorded at 5000 measurements/sec.

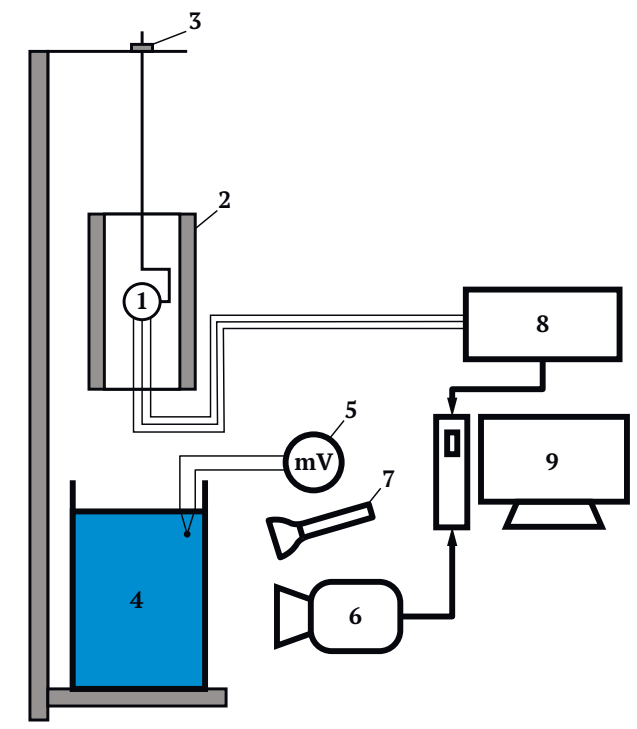

Figure 28. Experimental scheme: 1-testing sphere; 2-furnace; 3-holder; 4-tank; 5-Fluke 289 with thermocouple; 6-camera, Evercam-4-C; 7-light source; 8-measurement and computing system (MCS), NI PXI-1050; 9-PC.

Figure 29 shows a heat flux graph of the sphere cooling from the initial temperature of $300{ }^{\circ} \mathrm{C}$ to water saturation temperature. There is a noticeable difference between the heat flux in the north $\left(180^{\circ}\right)$ and south $\left(0^{\circ}\right)$ poles of the sphere. The first peaks on both curves correspond to the second critical heat flux; they range from $0.46 \mathrm{MW} / \mathrm{m}^{2}$ to $0.63 \mathrm{MW} / \mathrm{m}^{2}$.

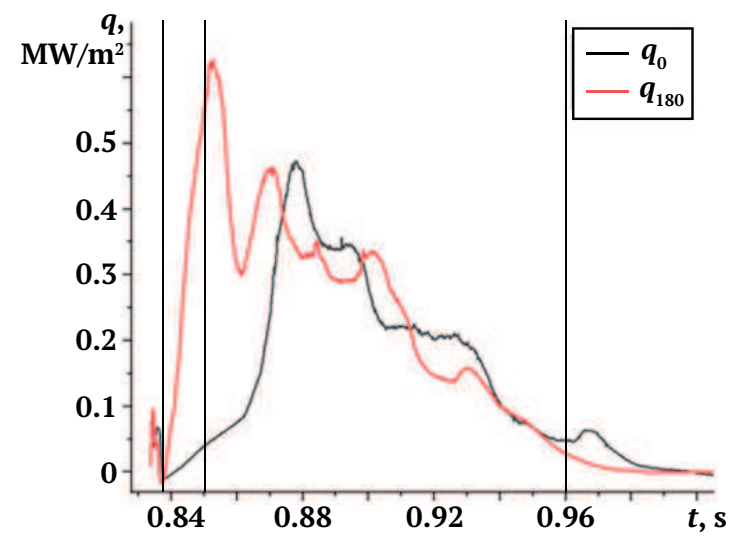

Figure 29. Dependence of heat flux on time: 1-film boiling; 2-boiling transition; 3-bubble boiling $\left(T_{w}=300^{\circ} \mathrm{C} ; T_{f}=100^{\circ} \mathrm{C}\right)$.

Then, we considered the heat transfer during subcooled liquid boiling [29,30]. We raised the temperature of the sphere and reduced the water temperature. Figure 30 shows as an example the 
results of experiments for a sphere with a temperature of $350{ }^{\circ} \mathrm{C}$, which was submerged in water with a temperature of $25^{\circ} \mathrm{C}$. A holder made it possible to rotate the ball around its horizontal axis, which gave the distribution of heat flux over the sphere surface. The experiment was repeated 12 times for the same sphere and water temperature, with the sensor rotation angles fixed every $15^{\circ}$.

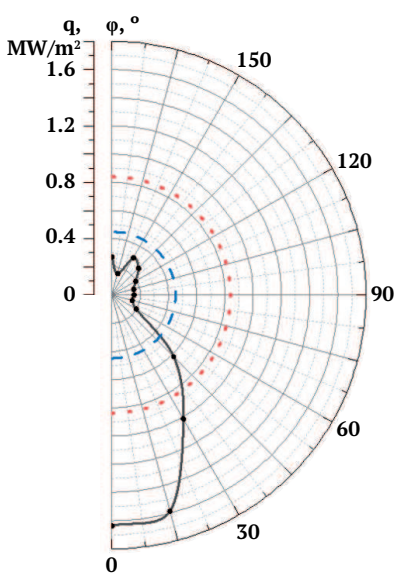

Figure 30. Angular thermal image $\left(T_{w}=350^{\circ} \mathrm{C} ; T_{f}=25^{\circ} \mathrm{C}\right)$.

No film boiling appeared on the surface of the ball in this experiment. The maximum heat flux was at an angle of $\varphi=0^{\circ}$. When the sphere cooled down, the heat flux was reduced by five times. The immersion photos are shown in Figure 31.

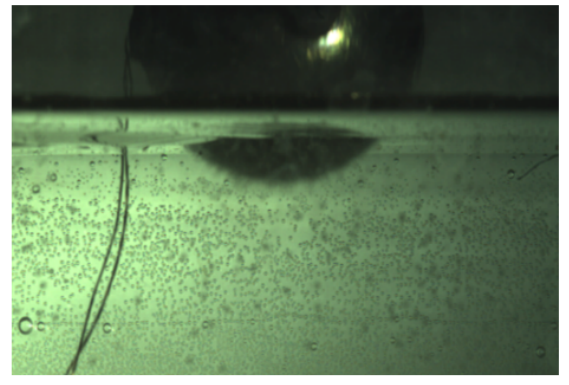

(a)

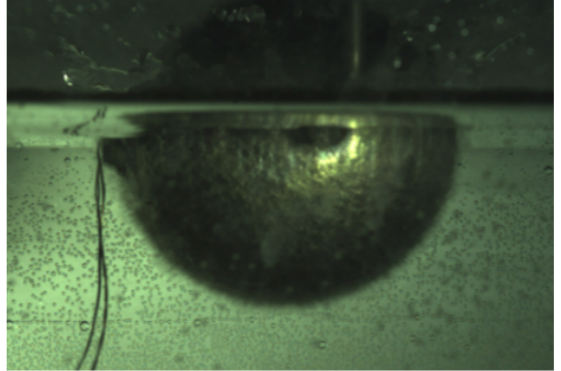

(b)

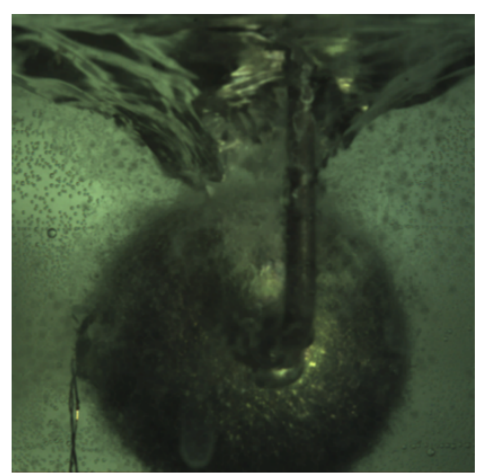

(c)

Figure 31. Sphere immersion photos: (a) start of immersion; (b) half-immersed sphere; (c) full-immersion. 
Average local heat flux by ball area is shown in Table 1.

Table 1. Experimental results.

\begin{tabular}{ccc}
\hline Mode & $\begin{array}{c}\text { Temperature Drop } \\
\mathbf{T}_{\boldsymbol{w}}-\mathbf{T}_{\boldsymbol{f}}, \mathbf{K}\end{array}$ & $\begin{array}{c}\text { Average Heat Flux } \\
\boldsymbol{q}, \mathbf{M W} / \mathbf{m}^{\mathbf{2}}\end{array}$ \\
\hline$T_{w}=450^{\circ} \mathrm{C} ; T_{f}=50^{\circ} \mathrm{C}$ & 400 & 9.332 \\
$T_{w}=450^{\circ} \mathrm{C} ; T_{f}=25^{\circ} \mathrm{C}$ & 425 & 3.929 \\
$T_{w}=350^{\circ} \mathrm{C} ; T_{f}=50^{\circ} \mathrm{C}$ & 300 & 0.930 \\
$T_{w}=350^{\circ} \mathrm{C} ; T_{f}=25^{\circ} \mathrm{C}$ & 325 & 0.241 \\
\hline
\end{tabular}

The results show that HTC does not depend on the initial temperature drop, but is mainly determined by water subcooling. This helps to select of coolant parameters in various technical applications. These studies are only the first step in the study of more complex non-isothermal surface boiling, including the finned surface.

\section{Conclusions}

The combination of gradient heatmetry with PIV and infrared imaging technologically enables a comprehensive study of heat transfer and flow near isothermal and non-isothermal surfaces. For the first time, measurements of velocity fields using PIV, infrared images, temperature measurement and gradient heatmetry were used simultaneously in one experiment, and the data obtained made it possible to analyze the processes of heat and mass transfer. A detailed study of the velocity fields and local heat transfer on the surface of a round cylinder was presented. Experiments made it possible to determine the optimal positions of the turbulizers on the cylinder's surface and increase the average heat transfer by $20 \%$. For two cylinders installed one after the other, the levels of heat flux fluctuations were determined for different flow regimes. For the first time, studies of heat transfer and flow near cylinders with ideal isothermal fins were carried out. Using gradient heatmetry, studies of local heat transfer during steam condensation at atmospheric pressure inside and outside tubes oriented in different ways in space have been carried out. The data on heat flux fluctuations along the tube perimeter were obtained. Gradient heatmetry made it possible to experimentally determine the local heat flux during boiling of a water on the surface of a heated model. Experimental data were obtained for saturated and subcooled water boiling on the surface of the sphere. The technique provides measurements in a non-stationary mode, and unlike high-speed visualization, makes it possible to reveal the relationship between the heat flux and the boiling mode. A number of scientific and industrial experiments confirmed the successful use of GHFS and their applicability.

Author Contributions: Conceptualization, S.Z.S. and V.Y.M.; methodology, S.Z.S., V.Y.M. and A.V.M.; validation, A.B.; formal analysis, A.Y.B.; investigation, V.V.S., A.A.G., A.V.P. and M.A.G.; data curation, E.R.Z.; writing-original draft preparation, V.V.S.; writing-review and editing, S.Z.S. and V.Y. M.; funding acquisition, A.A.G. All authors have read and agreed to the published version of the manuscript.

Funding: This research received no external funding.

Acknowledgments: This research was supported by the Academic Excellence Project 5-100 proposed by Peter the Great St. Petersburg Polytechnic University.

Conflicts of Interest: The authors declare no conflict of interest. 


\section{Abbreviations}

The following abbreviations are used in this manuscript:

$\begin{array}{ll}\text { HFS } & \text { Heat Flux Sensor } \\ \text { EMF } & \text { Electromotive Force } \\ \text { ATE } & \text { Anisotropic Thermoelemnt } \\ \text { GHFS } & \text { Gradient Heat Flux Sensor } \\ \text { HGHFS } & \text { Heterogeneous Gradient Heat Flux Sensor } \\ \text { PIV } & \text { Particle Image Velocimetry } \\ \text { HTC } & \text { Heat Transfer Coefficient } \\ \text { PSD } & \text { Poser Spectral Density } \\ \text { MCS } & \text { Measurement and Computing System }\end{array}$

\section{References}

1. Mityakov, V.; Seroshtanov, V.; Vlasov, A.; Suchok, V.; Bobylev, P.; Zhidkov, N. Heat transfer and air flow near a pair of circular cylinders. E3S Web Conf. 2019, 140, 06012. [CrossRef]

2. Sapozhnikov, S.; Mityakov, V.; Babich, A.; Zainullina, E. Study of condensation at the surfaces of tube with gradient heat flux measurement. MATEC Web Conf. 2018, 245, 06010. [CrossRef]

3. Sapozhnikov, S.; Mityakov, V.; Subbotina, V. Gradient heat flux measurement in study of unsteady water film boiling at the surface of the sphere. J. Phys. Conf. Ser. 2019, 1382, 012119. [CrossRef]

4. Goldstein, R.J.; Ibele, W.E.; Patankar, S.V.; Simon, T.W.; Kuehn, T.H.; Strykowski, P.J.; Tamma, K.K.; Heberlein, J.V.R.; Davidson, J.H.; Bischof, J.; et al. Heat transfer-A review of 2005 literature. Int. J. Heat Mass Transf. 2010, 53, 439-7-444-7. [CrossRef]

5. Available online: https://www.fluxteq.com/ (accessed on 3 January 2020).

6. Lartz, D.J.; Cudney, H.H.; Diller, T.E. Heat Flux Measurement and Heat Flux Sensor. In Heat Transfer; 1994; pp. 261-266. Available online: http:/ / www.ihtcdigitallibrary.com (accessed on 1 September 2020). [CrossRef]

7. Van der Graaf, F. Heat Flux Sensors. In Gopel (Ed.); “Thermal Sensors" of the Multivolume Work "Sensors, a Comprehensive Series"; Ricolfi, T., Scholz, J., Eds.; 1990; Chapter 8, Volume 4. Available online: https: / / onlinelibrary.wiley.com (accessed on 1 September 2020).

8. Divin, N.; Sapozhnikov, S.; Kirillov, A. Gradientenartige Messgeber fur die Messung des Warmestromes. In Proceedings Messtechnik zur Undersuchung von Vorgangen in Thermischen Energieanlagen. XXVIII. Kraftwerkstechnisches Kolloquium und 6. Kolloquium Messtechnik fur Energieanlagen; 1996; pp. $155-160$. Available online: https:/ /www.tib.eu/de/suchen/id/tema:TEMAM97031655556/Gradientenartige-Messgeberf\%C3\%BCr-die-Messung-des?cHash=df0ccaa1cbe4a920550c63f74291dff7/ (accessed on 1 September 2020).

9. US Patent and trade Mark Office Database. Available online: https://www.uspto.gov/ (accessed on 4 January 2020).

10. Anatychuk, L.I. Termoelementy i Termoelektricheskiye Ustroystva. Spravochnik (Thermoelements and Thermoelectric Devices. Refernce Book); Naukova Dumka: Kiev, Ukraine, 1979; p. 767.

11. Divin, N.P. Useful Model 9959 RF, “Heat flux sensor” May 16, 1999, Polez. Modeli. Prom. Obraztsy, No. 5. Available online: https:/ / rospatent.gov.ru (accessed on 10 July 2020)

12. Samoilovich, A.G. Termoelektricheskiye $i$ Termomagnitnyye metody Preobrazovaniya Energii (Thermoelectric and Thermomagnetic Methods of Energy Conversion); Mashinostroenie: Moscow, Russia, 1981.

13. Srinivasan, B.; Berthebaud, D.; Mori, T. Is LiI a Potential Dopant Candidate to Enhance the Thermoelectric Performance in Sb-Free GeTe Systems? A Prelusive Study. Energies 2020, 13, 643. [CrossRef]

14. Sapozhnikov, S.Z.; Mityakov, V.Y.; Mityakov, A.V. Heatmetry; Springer: Berlin/Heidelberg, Germany, $2020 ;$ p. 225.

15. Knauss, H.; Gaisbauer, U.; Wagner, S.; Buntin, D.; Maslov, A.; Smorodsky, B.; Betz, J. Calibration experiments of a new active fast response heat flux sensor to measure total temperature fluctuations. Part I Introduction to the problem. In Proceedings of the International Conference on the Methods of Aerophysical Research, Novosibirsk, Russia, 1-7 July 2002; pp. 632-643. 
16. ISO/IEC Guide 98-1:2009(en) Uncertainty of Measurement-Part 1: Introduction to the Expression of Uncertainty in Measurement. Available online: www.iso.org/standard (accessed on 5 September 2020).

17. Perrin, R.; Braza, M.; Cid, E.; Cazin, S.; Moradei, F.; Barthet, A.; Sevrain, A.; Hoarau, Y. Near-wake turbulence properties in the high Reynolds number incompressible flow around a circular cylinder measured by two- and three-component PIV. Flow Turbul. Combust 2006, 77, 185-204. [CrossRef]

18. Available online: http:/ / polis-instruments.ru/ (accessed on 1 September 2020).

19. Mikheev, N.; Molochnikov, V.; Mikheev, A.; Dushina, O. Hydrodynamics and heat transfer of pulsating flow around a cylinder. Int. J. Heat Mass Transf. 2017, 109, 254-265. [CrossRef]

20. Nakamura, H.; Igarashi, T. Unsteady heat transfer from a circular cylinder for Reynolds numbers from 3000 to 15,000. Int. J. Heat Fluid Flow 2004, 25, 741-748. [CrossRef]

21. Sahin, B.; Akkoca, A.; Ozturk, N.A.; Akilli, H. Investigations of flow characteristics in a plate fin and tube heat exchanger model composed of single cylinder. Int. J. Heat Fluid Flow 2006, 27, 522-530. [CrossRef]

22. Nada, S.A.; Hussein, M.S. General semi-empirical correlation for condensation of vapor on tubes at different orientations. Int. J. Therm. Sci. 2016, 100, 391-400. [CrossRef]

23. Ooi, T.H.; Webb, D.R.; Heggs, P.J. A dataset of steam condensation over a double enhanced tube bundle under vacuum. Appl. Therm. Eng. 2004, 24, 1381-1393. [CrossRef]

24. Sang, J.K.; Hee, C.N. Turbulent film condensation of high pressure steam in a vertical tube. Int. J. Heat Mass Transf. 2000, 43, 4031-4042.

25. Fan, G.; Tong, P.; Sun, Z.; Chen, Y. Development of a new empirical correlation for steam condensation rates in the presence of air outside vertical smooth tube. Ann. Nucl. Energy 2018, 113, 139-146. [CrossRef]

26. Chen, C.-K.; Lin, Y.-T. Laminar film condensation from a downward-flowing steam-air mixture onto a horizontal circular tube. Appl. Math. Model 2004, 33, 1944-1956. [CrossRef]

27. Saleh, E.A.; Ormiston, S.J. An elliptic two-phase numerical model of laminar film condensation from a steam-air mixture flowing over a horizontal tube. Int. J. Heat Mass Transf. 2004, 112, 676-688. [CrossRef]

28. Zabirov, A.R. Issledovaniye protsessov teploobmena pri okhlazhdenii vysokotemperaturnykh tel v nedogretykh zhidkostyakh (Investigation of heat transfer processes during cooling of high-temperature bodies in subcooled liquids). In Dissertation of a Candidate of Technical Sciences: Zabirov Arslan Ruslanovich; Moscow, Russia, 2016. Available online: www.dissercat.com (accessed on 1 August 2020).

29. Grigor'ev, V.S.; Zhilin, V.G.; Zeigarnik, Y.A.; Ivochkin, Y.P.; Glazkov, V.V.; Sinkevich, O.A. The Behavior of a Vapor Film on a Highly Superheated Surface Immersed in Subcooled Water. Heat Mass Transf. Phys. Gasdyn. 2005, 43, 100-114.

30. Gylys, J.; Skvorcinskiene, R.; Paukstaitis, L.; Gylys, M.; Adomavicius, A. Film boiling influence on the spherical body's cooling in sub-cooled water. Int. J. Heat Mass Transf. 2016, 95, 709-719. [CrossRef]

Publisher's Note: MDPI stays neutral with regard to jurisdictional claims in published maps and institutional affiliations.

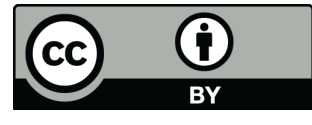

(C) 2020 by the authors. Licensee MDPI, Basel, Switzerland. This article is an open access article distributed under the terms and conditions of the Creative Commons Attribution (CC BY) license (http:/ / creativecommons.org/licenses/by/4.0/). 